\title{
Self-Oscillating Control Methods for the LCC Current-Output Resonant Converter
}

\author{
Adam J. Gilbert, Christopher M. Bingham, David A. Stone, and Martin P. Foster
}

\begin{abstract}
A strategy for self-oscillating control of LCC current-output resonant converters, is presented, based on varying the phase-angle between the fundamental of the input voltage and current. Unlike other commonly employed control methodologies, the proposed technique is shown to provide a convenient, linear system input-output characteristic suitable for the design of regulators. The method is shown to have a similar effect as controlling the dc-link supply voltage, in terms of output-voltage/current control. The LCC converter variant is used as an application focus for demonstrating the presented techniques, with simulation and experimental measurements from a prototype converter being used to show the practical benefits. Third-order small and large-signal models are developed, and employed in the formulation of robust output-voltage and output-current control schemes. However, notably, the presented techniques are ultimately generic and readily applicable to other resonant converter variants.
\end{abstract}

Index Terms-Zero current switching (ZCS), zero voltage switching (ZVS).

\section{INTRODUCTION}

$\mathbf{I}$ $\mathrm{T}$ is well established that resonant converter-based supplies are advantageous in-terms of size and efficiency [1], [2] compared to hard-switched counterparts. One of the main impediments to their use, however, is the lack of simple but robust control techniques. This is primarily due to the highly non-linear dynamic characteristics that are normally associated with such systems. A novel control strategy (based on a variant of linearized phase-control) is therefore proposed that approximately linearizes the behavior of the converter such that well-known control techniques can be readily applied. Although other methods have been previously reported [3], [4] that aim to linearize the converter's behavior, the mechanism presented here provides a convenient and cohesive framework that is widely applicable. To focus the development of the underlying principles, an LCC current-output converter variant, shown in Fig. 1, is used as an example.

The proposed techniques therefore constitute an advancement to commonly used phase-control methods, and maintains all the associated advantages over traditional frequency control methods. In [5], it is shown that self-oscillating phase-control provides self-tuning relative to the resonant tank frequency (advantageous in that the same phase-controller can be directly

Manuscript received August 24, 2007; revised February 6, 2008. Published July 7, 2008 (projected). Recommended for publication by Associate Editor J. M. Alonso.

The authors are with the Department of Electronic and Electrical Engineering, University of Sheffield, Sheffield S1 3JD, U.K. (e-mail: c.bingham@ sheffield.ac.uk).

Color versions of one or more of the figures in this paper are available online at http://ieeexplore.ieee.org.

Digital Object Identifier 10.1109/TPEL.2008.925198 applied to a wide range of converter designs), reduced controller sensitivity near resonance, and the ability to ensure switching at all times is either above resonance [for zero voltage switching (ZVS)] or below resonance [for zero current switching (ZCS)] depending on application requirements. To obtain the overall system dynamics when using the presented control methodology, similar analysis techniques are employed to those used in [6] for the frequency control of resonant-converters.

\section{EQUiVAlENCE OF POWER-FACTOR AND SUPPLY VOLTAGE CONTROL}

During normal operation, the resonant tank is excited above the resonant frequency by an applied square-wave voltage, $V_{s}$, that results in an approximately sinusoidal series inductor current, $I_{L s}$, see Fig. 2(a). The fundamental component of the square-wave is also shown in Fig. 2(a), and can be sub-divided into two components, one in phase with the resonant current, $I_{L s}$, and one in quadrature, Fig. 2(b).

The following identity can be used to derive the magnitude of the switching voltage component that is in-phase with the series inductor current:

$$
\sin (A+B)=\sin (A) \cos (B)+\cos (A) \sin (B) .
$$

The switching voltage can be approximated by its fundamental component as follows:

$$
V_{s}=\frac{V_{\mathrm{dc}}}{2} \operatorname{sgn}\left(\sin \left(\omega_{s} t\right)\right) \approx \frac{2 V_{\mathrm{dc}}}{\pi} \sin \left(\omega_{s} t\right)
$$

with the series inductor current assumed to be a sinusoid

$$
I_{L s}=\hat{I}_{L s} \sin \left(\omega_{s} t-\theta\right)
$$

where "^" denates the peak value, and is used generically throughout the following analysis.

Using (1) the fundamental component of the excitation voltage can be expressed as

$$
\begin{aligned}
\frac{2 V_{\mathrm{dc}}}{\pi} \sin \left(\omega_{s} t\right)=\frac{2 V_{\mathrm{dc}}}{\pi} \cos (\theta) & \sin \left(\omega_{s} t-\theta\right) \\
+ & \frac{2 V_{\mathrm{dc}}}{\pi} \sin (\theta) \cos \left(\omega_{s} t-\theta\right) .
\end{aligned}
$$

To maintain the real power flow into the converter, assuming a square-wave switching voltage in-phase with the series inductor current, the "equivalent" square-wave voltage is approximated to

$$
V_{s}=\frac{V_{\mathrm{dc}}}{2} \cos (\theta) \operatorname{sgn}\left(\sin \left(\omega_{s} t-\theta\right)\right)=\frac{V_{\mathrm{dc}} P F}{2} \operatorname{sgn}\left(\sin \left(\omega_{\mathrm{s}} t-\theta\right)\right)
$$

where the electrical power-factor $\mathrm{PF}=\cos (\theta)$ 


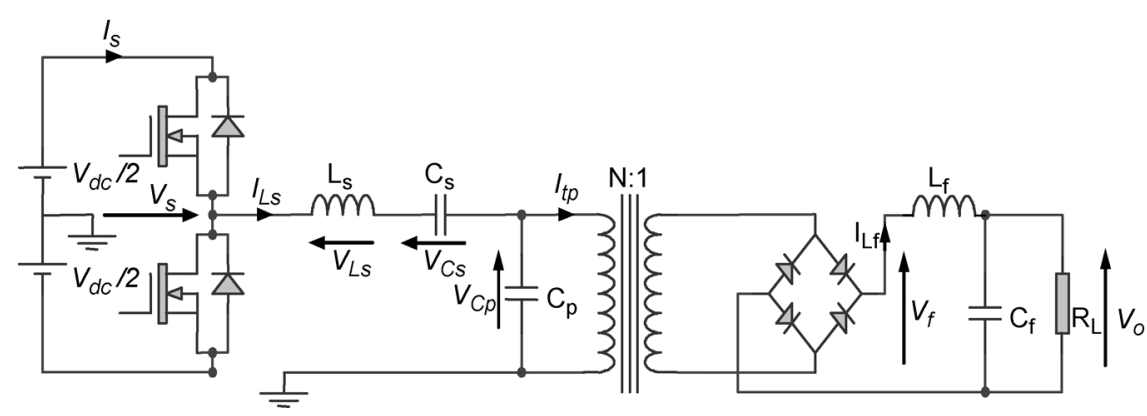

Fig. 1. LCC current-output resonant converter.

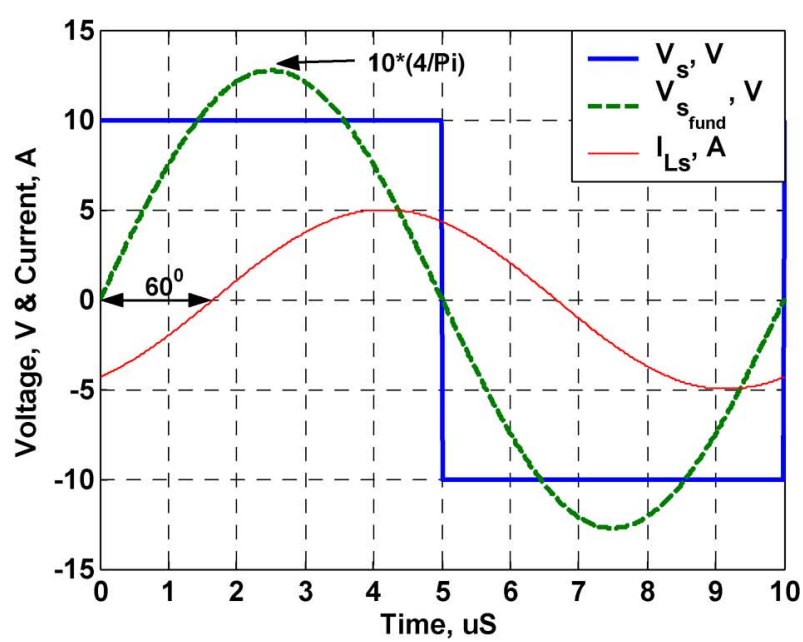

(a)

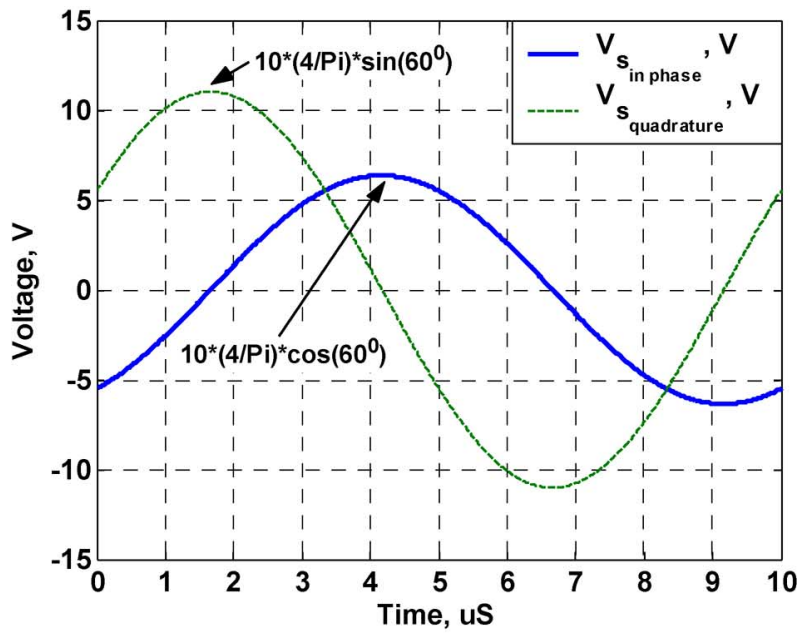

(b)

Fig. 2. (a) Excitation voltage and resonant tank current and (b) phase components of switching voltage wrt. resonant tank current.

It can now be seen that varying the instantaneous powerfactor $(P F)$ is equivalent to varying the instantaneous supply-voltage, from a fundamental mode approximation (FMA) perspective. When deriving a small-signal model, this allows the converter to be considered as being excited at the resonant frequency while varying the power factor-in this case, power-factor can be included in the model as an effective supply-voltage scaling factor, and therefore does not alter the structure of the model.

\section{PHASOR-TRANSFORM MODEL}

To enable a small-signal model of the self-oscillating power-factor controlled converter to be derived, a large-signal phasor-transformed model is initially considered when operating the converter about the resonant frequency. In a similar manner to the use of the $d-q$ axis transformation when modelling and analysing three-phase machines, a "phasor-transform" technique can be used for single-phase systems based on the assumption that the signals are sinusoidal in nature but vary in amplitude, frequency and phase [7]-[10]. These features are characteristic of resonant converters operating near resonance with sufficiently high quality factor, as is commonly encountered.

The formulation is based upon the principle that a generic form of sine waveform (voltages or currents in this case) can be approximated by a sinusoid whose frequency and amplitude vary with time [8], [9]

$$
x(t)=\Re\left(\bar{x}(t) e^{j \int_{0}^{t} \omega_{s}(t) d t}\right)
$$

where $\bar{x}(t)$ is the complex envelope of $x(t)$ and $\omega_{s}$ is the switching frequency. Considering the differential equation governing the behavior of an ideal inductor

$$
v(t)=L \frac{d i(t)}{d t}
$$

and substituting (6) into (7) for both current and voltage gives the generic expression

$$
\Re\left(\bar{v}(t) e^{j \int_{0}^{t} \omega_{s}(t) d t}\right)=L \frac{d}{d t} \Re\left(\bar{i}(t) e^{j \int_{0}^{t} \omega_{s}(t) d t}\right) .
$$

Simplifying gives

$$
\bar{v}(t)=L \frac{d \bar{i}(t)}{d t}+j L \omega_{s}(t) \bar{i}(t) .
$$

In a similar manner, capacitors and resistors are described by

$$
\begin{aligned}
\bar{i}(t) & =C \frac{d \bar{v}(t)}{d t}+j C \omega_{s}(t) \bar{v}(t) \\
\bar{v}(t) & =R \bar{i}(t) .
\end{aligned}
$$

More generally, therefore, a signal envelope $\bar{x}(t)$ can be expanded into its constituent real and imaginary components, denoted by " $r$ " and " $i$ " subscripts, respectively

$$
\bar{x}(t)=x_{r}(t)+j x_{i}(t) .
$$




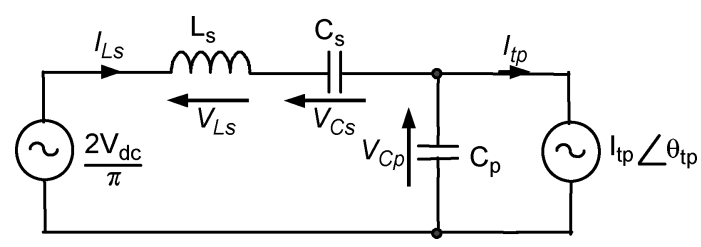

Fig. 3. FMA model of LCC resonant tank.

For the inductor, substituting (11) into (9) and separating the real and imaginary terms gives

$$
\begin{aligned}
& v_{r}(t)=L \frac{d i_{r}(t)}{d t}-L \omega_{s}(t) i_{i}(t) \\
& v_{i}(t)=L \frac{d i_{i}(t)}{d t}+L \omega_{s}(t) i_{r}(t)
\end{aligned}
$$

and similarly for the capacitor and resistor

$$
\begin{aligned}
i_{r}(t) & =C \frac{d v_{r}(t)}{d t}-C \omega_{s}(t) v_{i}(t) \\
i_{i}(t) & =C \frac{d v_{i}(t)}{d t}+C \omega_{s}(t) v_{r}(t) \\
v_{r}(t) & =R i_{r}(t) \\
v_{i}(t) & =R i_{i}(t) .
\end{aligned}
$$

The rectifier in Fig. 1 is not readily modelled using the phasortransform technique, and requires the resonant tank and output filter to be considered separately, and then combined. This is justified since the output filter bandwidth is much lower than that of the tank resonant frequency, and, as a result of the rectifier action, the filter effectively reacts only to the envelope of the resonant tank waveforms. From basic FMA, the resonant tank is therefore modelled as in Fig. 3 [2].

For use in network simulators (such as SPICE or SABER), the complex components can be eliminated through use of two coupled equivalent circuits, describing separately, the real and imaginary characteristics [10]. After phasor-transforming the components in Fig. 3, the coupled networks shown in Fig. 4 are obtained.

A state space representation of Fig. 4 is obtained for both the real and imaginary circuits, which are then combined to give (15)

$$
\begin{gathered}
{\left[\begin{array}{c}
\dot{I}_{L s_{r}} \\
\dot{V}_{C s_{r}} \\
\dot{V}_{C p_{r}} \\
\dot{I}_{L s_{i}} \\
\dot{V}_{C s_{i}} \\
\dot{V}_{C p_{i}}
\end{array}\right]=\left[\begin{array}{cccccc}
0 & \frac{-1}{L_{s}} & \frac{-1}{L_{s}} & \omega_{s} & 0 & 0 \\
\frac{1}{C_{s}} & 0 & 0 & 0 & \omega_{s} & 0 \\
\frac{1}{C_{p}} & 0 & 0 & 0 & 0 & \omega_{s} \\
-\omega_{s} & 0 & 0 & 0 & \frac{-1}{L_{s}} & \frac{-1}{L_{s}} \\
0 & -\omega_{s} & 0 & \frac{1}{C_{s}} & 0 & 0 \\
0 & 0 & -\omega_{s} & \frac{1}{C_{p}} & 0 & 0
\end{array}\right]\left[\begin{array}{c}
I_{L s_{r}} \\
V_{C s_{r}} \\
V_{C p_{r}} \\
I_{L s_{i}} \\
V_{C s_{i}} \\
V_{C p_{i}}
\end{array}\right]} \\
+\left[\begin{array}{ccc}
\frac{2}{\pi L_{s}} & 0 & 0 \\
0 & 0 & 0 \\
0 & \frac{-1}{C_{p}} & 0 \\
0 & 0 & 0 \\
0 & 0 & 0 \\
0 & 0 & \frac{-1}{C_{p}}
\end{array}\right]\left[\begin{array}{c}
V_{\mathrm{dc}} \\
I_{t p_{r}} \\
I_{t p_{i}}
\end{array}\right]
\end{gathered}
$$

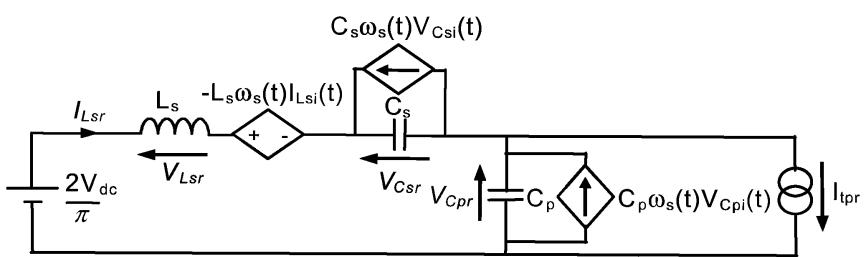

(a)

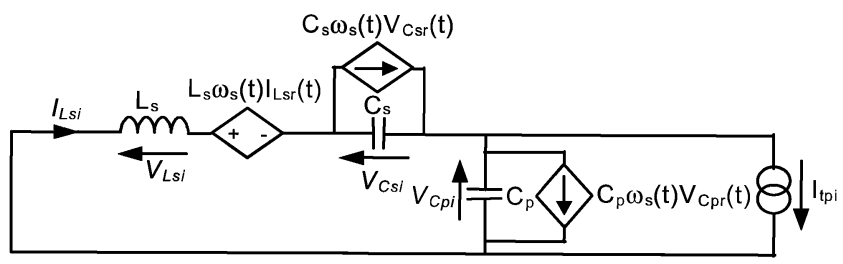

(b)

Fig. 4. Phasor transformed LCC resonant tank model: (a) real circuit and (b) imaginary circuit.

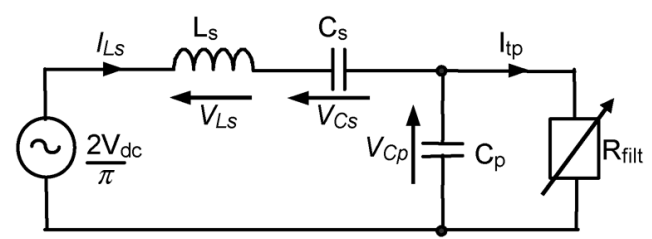

Fig. 5. Modelling output filter by a time varying load.

and the waveform envelopes are extracted using the output equation

$$
\left[\begin{array}{c}
\hat{I}_{L s} \\
\hat{V}_{C r} \\
\hat{V}_{C s}
\end{array}\right]=\left|\left[\begin{array}{llllll}
1 & 0 & 0 & j & 0 & 0 \\
0 & 1 & 0 & 0 & j & 0 \\
0 & 0 & 1 & 0 & 0 & j
\end{array}\right]\left[\begin{array}{c}
I_{L s_{r}} \\
V_{C s_{r}} \\
V_{C p_{r}} \\
I_{L s_{i}} \\
V_{C s_{i}} \\
V_{C p_{i}}
\end{array}\right]\right| .
$$

Finally, the output filter is described in state-variable form by

$$
\left[\begin{array}{c}
\dot{I}_{L f} \\
\dot{V}_{o}
\end{array}\right]=\left[\begin{array}{cc}
0 & \frac{-1}{L_{f}} \\
\frac{1}{C_{f}} & \frac{-1}{R_{L} C_{f}}
\end{array}\right]\left[\begin{array}{c}
I_{L f} \\
V_{o}
\end{array}\right]+\left[\begin{array}{c}
\frac{1}{L_{f}} \\
0
\end{array}\right]\left[V_{f}\right] .
$$

The voltages of the phasor-transformed tank model and output filter are coupled by noting that the average voltage presented to the output filter, via the rectifier, is given by (18), since $V_{C p}$ is assumed sinusoidal

$$
V_{f}=\frac{2 \hat{V}_{C p}}{N \pi} .
$$

Coupling of the currents is achieved by replacing the current source in Fig. 3 by a time varying resistor, Fig. 5, with the imaginary and real components of $I_{t p}$ given by

$$
I_{t p_{r}}=\frac{V_{C p_{r}}}{R_{f i l t}}, \quad I_{t p_{i}}=\frac{V_{C p_{i}}}{R_{f i l t}} .
$$


Since the transformer primary current is a squarewave, and the fundamental is used in the phasor-transformed model, the peak of the squarewave current is scaled by $4 / \pi$ :

$$
\hat{I}_{t p}=\sqrt{I_{t p_{r}}^{2}+I_{t p_{i}}^{2}}=\frac{4 I_{L f}}{N \pi} .
$$

The time-varying load resistance is now calculated from

$$
R_{f i l t}=\frac{\hat{V}_{C p}}{\hat{I}_{t p}}
$$

From (19), (20) and (21) the transformer primary phasor currents are found algebraically

$$
I_{t p_{r}}=\frac{4 I_{L f}}{N \pi} \frac{V_{C p_{r}}}{\hat{V}_{C p}}, \quad I_{t p_{i}}=\frac{4 I_{L f}}{N \pi} \frac{V_{C p_{i}}}{\hat{V}_{C p}} .
$$

The complete model is therefore described by (15)-(18) and (22).

\section{Small-Signal AnAlysis}

A small-signal model of the converter, while under self-oscillating power-factor control, can be obtained by modifying (15) (the large signal phasor-transform model) such that power factor becomes an input. In Section II it has been shown that varying the power-factor is equivalent in terms of real power flow to linearly scaling the supply voltage while switching the converter at resonance, hence

$$
\begin{gathered}
{\left[\begin{array}{c}
\dot{I}_{L s_{r}} \\
\dot{V}_{C s_{r}} \\
\dot{V}_{C p_{r}} \\
\dot{I}_{L s_{i}} \\
\dot{V}_{C s_{i}} \\
\dot{V}_{C p_{i}}
\end{array}\right]=\left[\begin{array}{cccccc}
0 & \frac{-1}{L_{s}} & \frac{-1}{L_{s}} & \omega_{r} & 0 & 0 \\
\frac{1}{C_{s}} & 0 & 0 & 0 & \omega_{r} & 0 \\
\frac{1}{C_{p}} & 0 & 0 & 0 & 0 & \omega_{r} \\
-\omega_{r} & 0 & 0 & 0 & \frac{-1}{L_{s}} & \frac{-1}{L_{s}} \\
0 & -\omega_{r} & 0 & \frac{1}{C_{s}} & 0 & 0 \\
0 & 0 & -\omega_{r} & \frac{1}{C_{p}} & 0 & 0
\end{array}\right]\left[\begin{array}{l}
I_{L s_{r}} \\
V_{C s_{r}} \\
V_{C p_{r}} \\
I_{L s_{i}} \\
V_{C s_{i}} \\
V_{C p_{i}}
\end{array}\right]} \\
+\left[\begin{array}{ccc}
\frac{2 V_{\mathrm{dc}}}{\pi L_{s}} & 0 & 0 \\
0 & 0 & 0 \\
0 & \frac{-1}{C_{p}} & 0 \\
0 & 0 & 0 \\
0 & 0 & 0 \\
0 & 0 & \overline{-1}
\end{array}\right]\left[\begin{array}{l}
P F \\
I_{t p_{r}} \\
I_{t p_{i}}
\end{array}\right] .
\end{gathered}
$$

[Note: When varying the power factor, the switching frequency naturally varies, and from an analysis perspective, either the supply voltage can be considered constant and the frequency varied, or, as in the case presented here, the supply voltage varied while the switching frequency remains constant]

The equations underpinning the phasor-transformed model (15)-(18), (22) are linearized about the resonant frequency, and (18) is re-expressed as follows:

$$
V_{f}=\frac{2 \sqrt{V_{C p_{r}}^{2}+V_{C p_{i}}^{2}}}{N \pi} .
$$

For a generic variable $X(t)$, the steady-state and small-signal components are separated as follows:

$$
X=X_{o}+\Delta x
$$

where $X_{o}$ and $\Delta x$ are the steady-state and small-signal components, respectively - and (24) is re-written as

$$
V_{f}=\frac{2 \sqrt{\left(V_{C p_{r} o}+\Delta v_{C p_{r}}\right)^{2}+\left(V_{C p_{i} o}+\Delta v_{C p_{i}}\right)^{2}}}{N \pi} .
$$

Linearizing about a steady-state operating point gives

$$
\Delta v_{f}=\frac{2}{N \pi \sqrt{V_{C p_{r} o}^{2}+V_{C p_{i} o}^{2}}}\left(V_{C p_{r} o} \Delta v_{C p_{r}}+V_{C p_{i} o} \Delta v_{C p_{i}}\right) \text {. }
$$

The transformer primary currents (22) are also modified accordingly

$$
\begin{aligned}
& I_{t p_{r}}=\frac{4}{N \pi} \frac{\left(I_{L f_{o}}+\Delta I_{L f}\right)\left(V_{C p_{r 0}}+\Delta v_{C p_{r}}\right)}{\sqrt{\left(V_{C p_{r 0}}+\Delta v_{C p_{r}}\right)^{2}+\left(V_{C p_{i 0}}+\Delta v_{C p_{i}}\right)^{2}}} \\
& I_{t p_{i}}=\frac{4}{N \pi} \frac{\left(I_{L f_{0}}+\Delta I_{L f}\right)\left(V_{C p_{i 0}}+\Delta v_{C p_{i}}\right)}{\sqrt{\left(V_{C p_{r 0}}+\Delta v_{C p_{r}}\right)^{2}+\left(V_{C p_{i 0}}+\Delta v_{C p_{i}}\right)^{2}}}
\end{aligned}
$$

and linearizing (28) about the steady-state operating point gives

$$
\begin{aligned}
\Delta i_{t p_{r}}= & \frac{4 I_{L f_{0}} V_{C p_{i 0}}^{2} \Delta v_{C p_{r}}}{N \pi\left(V_{C p_{r 0}}^{2}+V_{C p_{i 0}}^{2}\right)^{(3 / 2)}} \\
& -\frac{4 I_{L f_{o}} V_{C p_{i 0}} V_{C p_{r 0}} \Delta v_{C p_{i}}}{N \pi\left(V_{C p_{r 0}}^{2}+V_{C p_{i 0}}^{2}\right)^{(3 / 2)}} \\
& +\frac{4 V_{C p_{r 0}} \Delta i_{L f}}{N \pi \sqrt{V_{C p_{r 0}}^{2}+V_{C p_{i 0}}^{2}}} \\
= & \frac{4 I_{L f_{0}} V_{C p_{r 0}}^{2} \Delta v_{C p_{i}}}{N \pi\left(V_{C p_{r 0}}^{2}+V_{C p_{i 0}}^{2}\right)^{(3 / 2)}} \\
& -\frac{4 I_{L f_{o}} V_{C p_{i 0}} V_{C p_{r 0}} \Delta v_{C p_{r}}}{N \pi\left(V_{C p_{r 0}}^{2}+V_{C p_{i 0}}^{2}\right)^{(3 / 2)}} \\
& +\frac{4 V_{C p_{i 0}} \Delta i_{L f}}{N \pi \sqrt{V_{C p_{r 0}}^{2}+V_{C p_{i 0}}^{2}}} .
\end{aligned}
$$

To develop the small-signal model, expressions for the steady-state components $V_{C p_{r 0}}, V_{C p_{i 0}}$ and $I_{L f_{0}}$ need to be derived. Using (24), the steady-state output inductor current is simply written

$$
I_{L f_{0}}=\frac{2 \sqrt{V_{C p_{r 0}}^{2}+V_{C p_{i 0}}^{2}}}{N \pi R_{L}}
$$

with the transformer primary current components, from (22) and (30), becoming

$$
\begin{aligned}
& I_{t p_{r}}=\frac{4 I_{L f}}{N \pi} \frac{V_{C p_{r}}}{\sqrt{V_{C p_{r}}^{2}+V_{C p_{i}}^{2}}}=\frac{8 V_{C p_{r}}}{N^{2} \pi^{2} R_{L}}, \\
& I_{t p_{i}}=\frac{4 I_{L f}}{N \pi} \frac{V_{C p_{i}}}{\sqrt{V_{C p_{r}}^{2}+V_{C p_{i}}^{2}}}=\frac{8 V_{C p_{i}}}{N^{2} \pi^{2} R_{L}} .
\end{aligned}
$$


Steady-state conditions are obtained by equating the time derivatives in (23) to zero. Equation (31) is used to replace the $I_{t p}$ components, thereby giving

$$
\begin{aligned}
\dot{I}_{L s_{r 0}}=0 & =-\frac{1}{L_{s}} V_{C s_{r 0}}-\frac{1}{L_{s}} V_{C p_{r 0}}+\omega_{s} I_{L s_{i 0}} \\
& +\frac{2 V_{\mathrm{dc}}}{\pi L_{s}} P F \\
\dot{V}_{C s_{r 0}}= & 0=\frac{1}{C_{s}} I_{L s_{r 0}}+\omega_{s} V_{C s_{i 0}} \\
\dot{V}_{C p_{r 0}}=0 & =\frac{1}{C_{p}} I_{L s_{r 0}}+\omega_{s} V_{C p_{i 0}}-\frac{1}{C_{p}}\left(\frac{8 V_{C p_{r 0}}}{N^{2} \pi^{2} R_{L}}\right) \\
\dot{I}_{L s_{i 0}}=0 & =-\frac{1}{L_{s}} V_{C s_{i 0}}-\frac{1}{L_{s}} V_{C p_{i 0}}-\omega_{s} I_{L s_{r 0}} \\
\dot{V}_{C s_{i 0}}= & =\frac{1}{C_{s}} I_{L s_{i 0}}-\omega_{s} V_{C s_{r 0}} \\
\dot{V}_{C p_{i 0}}=0 & =\frac{1}{C_{p}} I_{L s_{i 0}}-\omega_{s} V_{C p_{r 0}}+-\frac{1}{C_{p}}\left(\frac{8 V_{C p_{i 0}}}{N^{2} \pi^{2} R_{L}}\right) .
\end{aligned}
$$

As previously shown, the power factor, in steady-state, does not alter the small-signal analysis. Consequently, it can be assumed that $P F=1$ (i.e., operation at resonance) to simplify the analysis. At resonance, therefore, $I_{L s_{i 0}}=0$, since all the series-inductor current is real. From (32e) this also implies that $V_{C s_{r 0}}=0$. Substituting $I_{L s_{i 0}}=0$ and $V_{C s_{r 0}}=0$ into (32a) allows for the calculation of $V_{C p_{r 0}}$

$$
V_{C p_{r 0}}=\frac{2 V_{\mathrm{dc}}}{\pi} \text {. }
$$

From the account given in [11], design equations for the LCC current-output resonant converter, based on FMA, can be utilized to simplify the analysis. Specifically, in [11], it is shown that, at resonance, the output voltage of the converter is given by:

$$
V_{o}=\frac{V_{\mathrm{dc}} G_{t r}}{N}
$$

where $G_{t r}$ is the voltage gain of the resonant tank and $N$ is transformer turns ratio (when expressed as $N: 1$ ), and the steady-state output-filter current is given by

$$
I_{L f_{0}}=\frac{V_{\mathrm{dc}} G_{t r}}{N R_{L}} .
$$

Equating (30) and (35), eliminating $V_{C p_{r 0}}$ via use of (33), and solving for $V_{C p_{i 0}}$ then gives

$$
V_{C p_{i 0}}=-\frac{V_{\mathrm{dc}} \sqrt{G_{t r}^{2} \pi^{4}-16}}{2 \pi} .
$$

Note that two solutions exist for (36), having opposing polarities. The solution presented in (36) is selected such that the phase of $V_{C p}$ w.r.t $I_{L s}$, is negative, i.e., $\arctan \left(V_{C p_{i 0}} / V_{C p_{r 0}}\right)<0$. In [11] it is also shown that, from a given converter specification, the resonant tank components can be chosen as follows:

$$
C_{p}=\frac{2 \sqrt{G_{t r}^{2} \pi^{4}-16}}{\pi^{2} N^{2} R_{L} \omega_{r}}
$$

$$
\begin{aligned}
C_{s} & =\frac{2 \sqrt{G_{t t}^{2} \pi^{4}-16}}{A \pi^{2} N^{2} R_{L} \omega_{r}} \\
L_{s} & =\frac{N^{2} R_{L}\left((A+1) G_{t r}^{2} \pi^{4}-16\right)}{2 \pi^{2} \omega_{r} G_{t r}^{2} \sqrt{G_{t r}^{2} \pi^{4}-16}}
\end{aligned}
$$

where $A=C_{p} / C_{s}$, and $\omega_{r}$ is the resonant frequency.

These equations will be used to simplify the remaining analysis. Substituting (33) and (36), into (32c), and eliminating $C_{p}$ in $(32 \mathrm{c})$ via (37a) gives

$$
I_{L s_{r 0}}=\frac{V_{\mathrm{dc}} G_{t r}^{2} \pi}{N^{2} R_{L}} .
$$

Eliminating $C_{s}$ in (32b) via (37b), substituting in (38) and solving for $V_{C s_{i 0}}$ then gives

$$
V_{C s_{i 0}}=-\frac{A V_{\mathrm{dc}} G_{t r}^{2} \pi^{3}}{2 \sqrt{G_{t r}^{2} \pi^{4}-16}} .
$$

Since the steady-state conditions are all now known (at PF $=1$ ) the small-signal equations expressed in (27), (29) can be determined

$$
\begin{aligned}
\Delta v_{f}= & \frac{2}{N \pi^{3} G_{t r}}\left(4 \Delta v_{C p_{r}}-\sqrt{G_{t r}^{2} \pi^{4}-16} \Delta v_{C p_{i}}\right) \\
\Delta i_{t p_{r}}= & \frac{8}{\pi^{2} N^{2} R_{L}}\left(1-\frac{16}{G_{t r}^{2} \pi^{4}}\right) \Delta v_{C p_{r}} \\
& +\frac{32 \sqrt{G_{t r}^{2} \pi^{4}-16}}{G_{t r}^{2} \pi^{6} N^{2} R_{L}} \Delta v_{C p_{i}}+\frac{16}{G_{t r} \pi^{3} N} \Delta i_{L f} \\
\Delta i_{t p_{r}}= & \frac{32 \sqrt{G_{t r}^{2} \pi^{4}-16}}{G_{t r}^{2} \pi^{6} N^{2} R_{L}} \Delta v_{C p_{r}}+\frac{128}{G_{t r}^{2} \pi^{6} N^{2} R_{L}} \Delta v_{C p_{i}} \\
& -\frac{4 \sqrt{G_{t r}^{2} \pi^{4}-16}}{G_{t r} \pi^{3} N} \Delta i_{L f} .
\end{aligned}
$$

The resulting state-space small-signal model can now be written from (17), (23), (40) and (41) giving (42), shown at the bottom of the next page.

To use the model, (42), the converter tank gain and resonant frequency must be determined. These are obtained by solving (37) for $A, G_{t r}$ and $\omega_{r}$. For instance, to calculate $G_{t r}$, equate the $\omega_{r}$ term in both (37a) and (37c), and solve for $G_{t r}$, where $A=C_{p} / C_{s}$.

\section{REAlization of Self-Oscillating CONTROLleR}

A key factor to provide good self-oscillating control of the power factor is determining when changes of polarity of the series-inductor current occur-for simplicity, the series-inductor voltage is measured, integrated (through use of low-pass filter with relatively low break frequency), and fed into low-offset comparator with an appropriate reference. The time-constant of the low-pass filter is chosen such that at the lowest switching frequency, the phase shift, $\theta$, is approximately $-\pi / 2$ and that the filter gain $G$ is sufficiently small such that the voltage applied across the comparator is within specification at all times

$$
\begin{aligned}
\theta & =-\tan ^{-1}(2 \pi f C R) \approx-\frac{\pi}{2} \\
G & =\frac{1}{\sqrt{1+(2 \pi f C R)^{2}}} .
\end{aligned}
$$




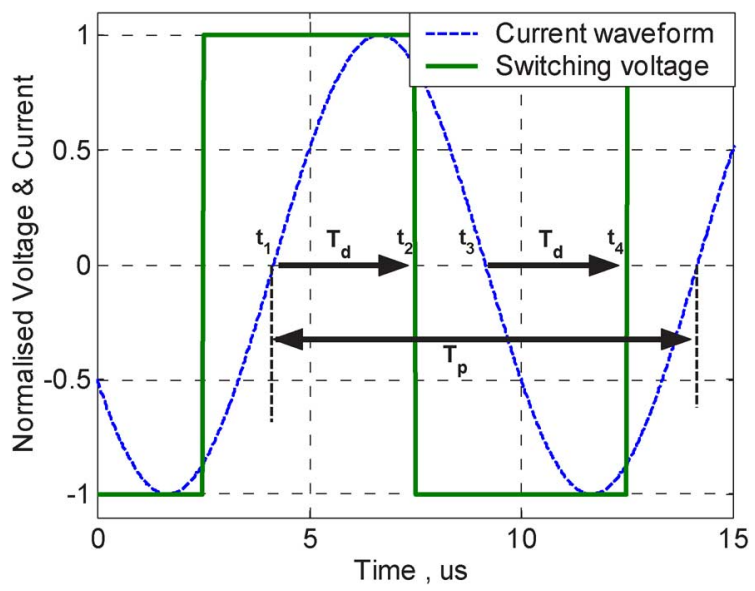

Fig. 6. FPGA delay methodology.

Having obtained the current polarity, an FPGA is used to generate the required switching signals based on the demanded power-factor. Fig. 6 shows the required functionality of the FPGA delay mechanism. A background process on the FPGA measures the period $\left(T_{p}\right)$ of the current waveform. At time $t_{1}$ a rising edge on $\operatorname{sgn}\left(I_{L s}\right)$ is detected, and, after a delay time $\left(T_{d}\right)$, at $t_{2}$, the FPGA forces a falling-edge on the switching voltage.

Using the methodology outlined above, the FPGA ensures the phase-shift is as desired on a cycle-by-cycle basis. The resulting large-signal model approximation relating power-factor to output-voltage is described in (42).

Delaying the "edges" in the proposed manner reduces the delays necessary by the FPGA and, secondly, ensures that the duty cycle of the current-waveform is maintained.

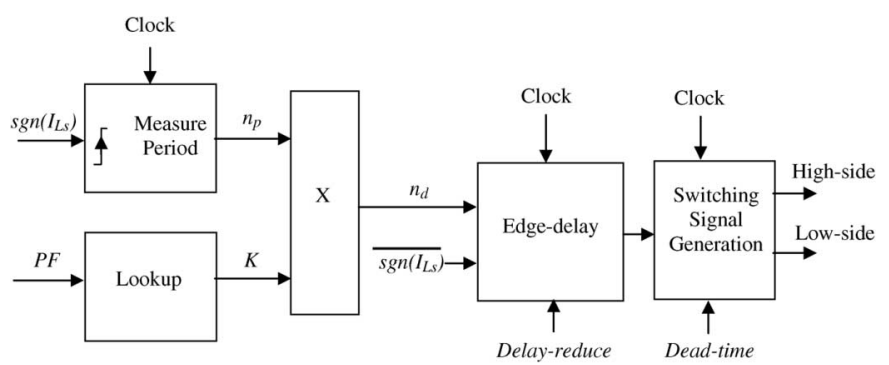

(a)

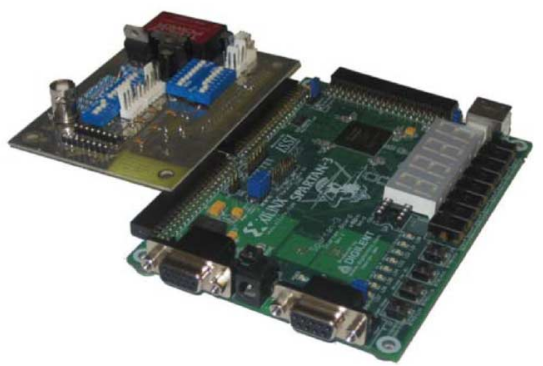

(b)

Fig. 7. FPGA based power-factor controller: (a) block diagram and (b) prototype controller.

The required delay-time $T_{d}$ is calculated from the powerfactor $(P F)$ and the period $T_{p}$ as follows:

$$
T_{d}=\frac{T_{p}}{2}\left(1-\frac{\cos ^{-1}(P F)}{\pi}\right) .
$$

To ensure self-oscillation start-up, when no series-inductor current sign change is detected within $100 \mu \mathrm{s}$, the FPGA switches the converter at a fixed $500 \mathrm{kHz}$. For the prototype presented in Section VI, this start-up strategy results in the

$$
\begin{aligned}
& A=\left[\begin{array}{cccccccc}
0 & -1 / L_{s} & -1 / L_{s} & \omega_{r} & 0 & 0 & 0 & 0 \\
1 / C_{s} & 0 & 0 & 0 & \omega_{r} & 0 & 0 & 0 \\
1 / C_{p} & 0 & K_{33} & 0 & 0 & \left(\omega_{r}+K_{36}\right) & K_{37} & 0 \\
-\omega_{r} & 0 & 0 & 0 & -1 / L_{s} & -1 / L_{s} & 0 & 0 \\
0 & -\omega_{r} & 0 & 1 / C_{s} & 0 & 0 & 0 & 0 \\
0 & 0 & \left(-\omega_{r}+K_{63}\right) & 1 / C_{p} & 0 & K_{66} & K_{67} & 0 \\
0 & 0 & K_{73} & 0 & 0 & K_{76} & 0 & -1 / L_{f} \\
0 & 0 & 0 & 0 & 0 & 0 & 1 / C_{f} & -1 /\left(C_{f} R_{L}\right)
\end{array}\right]
\end{aligned}
$$

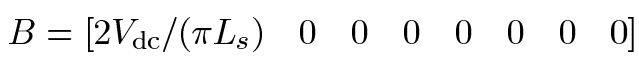

$$
\begin{aligned}
& C=\left[\begin{array}{llllllll}
0 & 0 & 0 & 0 & 0 & 0 & 0 & 1
\end{array}\right] \\
& x=\left[\Delta I_{L s_{r}} \Delta V_{C s_{r}} \Delta V_{C p_{r}} \Delta I_{L s_{i}} \Delta V_{C s_{i}} \Delta V_{C p_{i}} \Delta I_{L f} \Delta V_{o}\right]^{T} \\
& \dot{x}=A \cdot x+B \cdot u \\
& y=C \cdot x \quad \text { where } y=\Delta V_{o} \text { and } u=\Delta P F \text {, } \\
& K_{33}=-\frac{4 \omega_{r} \sqrt{G_{t r}^{2} \pi^{4}-16}}{G_{t r}^{2} \pi^{4}} \quad K_{36}=-\frac{16 \omega_{r}}{G_{t r}^{2} \pi^{4}} \quad K_{37}=-\frac{8 N R_{L} \omega_{r}}{G_{t r} \pi \sqrt{G_{t r}^{2} \pi^{4}-16}} \\
& K_{63}=-\frac{16 \omega_{r}}{G_{t r}^{2} \pi^{4}} \quad K_{66}=-\frac{64 \omega_{r}}{G_{t r}^{2} \pi^{4} \sqrt{G_{t r}^{2} \pi^{4}-16}} \quad K_{67}=\frac{2 N R_{L} \omega_{r}}{G_{t r} \pi} \\
& K_{73}=\frac{8}{L_{f} G_{t r} \pi^{3} N} \quad K_{76}=\frac{-2 \sqrt{G_{t r}^{2} \pi^{4}-16}}{L_{f} G_{t r} \pi^{3} N}
\end{aligned}
$$




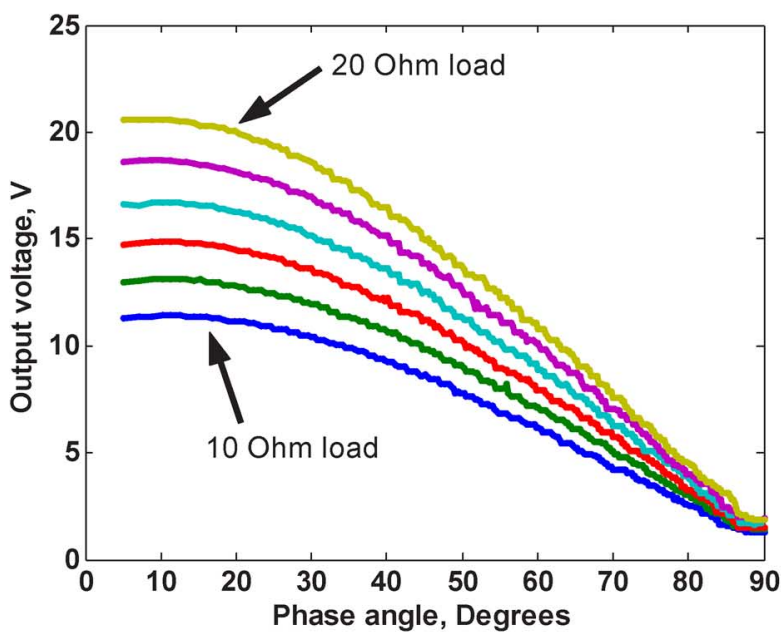

(a)

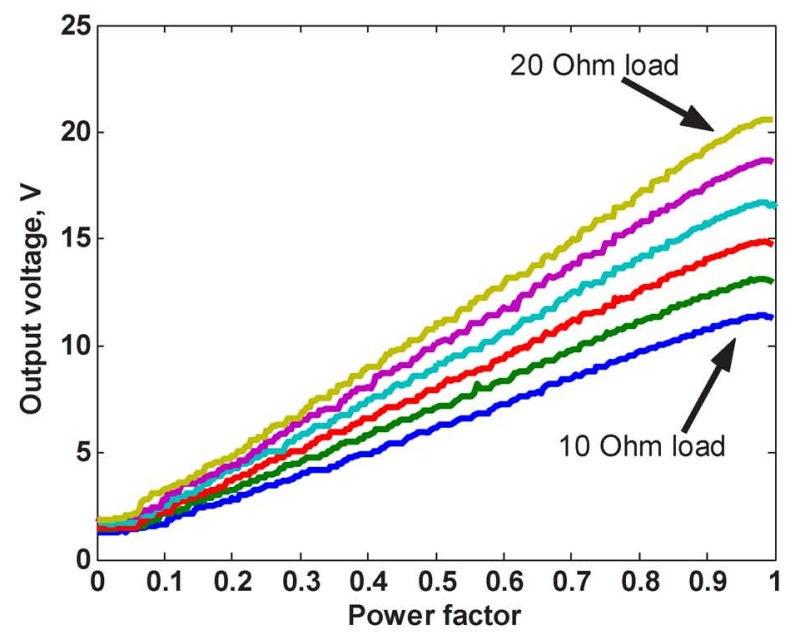

(b)

Fig. 8. Steady-state output voltage across various loads when using (a) phase-angle control and (b) power-factor control.

converter being switched at $500 \mathrm{kHz}$ for approximately $10 \mu \mathrm{s}$ before the series-inductor current is sufficient to start self-oscillation. The technique additionally results in reduced inrush currents.

An overview of the power factor controller, and photo of the FPGA with interface board, is provided in Fig. 7.

The delay-reduce feature provides compensation for any propagation delay in current sensing, processing, and gate driving. It should be noted that from a commercial perspective, a fully embedded closed-loop output-regulation control strategy suitable for low-cost and robust resonant-converter control, would be implemented on a low cost ASIC.

\section{Steady-State Behavior of the POWER-FACTOR CONTROLLER}

For a 1-A current-source when switched at the resonant frequency [12] with converter parameters specified by: $V_{\mathrm{dc}}=18$, $L_{s}=13.6 \mu \mathrm{H}, C_{s}=220 \mathrm{nF}, C_{p}=130 \mathrm{nF}, N=1, L_{f}=$ $24 \mu \mathrm{H}, C_{f}=220 \mu \mathrm{F}$, Fig. 8 provides a comparison between experimental measurements of steady-state output voltage for both the proposed self-oscillating power-factor control methodology and the more traditional self-oscillating phase-angle control methodology, as the load is varied from 10 to $20 \Omega$ in $2 \Omega$ increments.

From Fig. 8 it can clearly be seen that, at steady-state, the power-factor control methodology provides an almost linear control to output relationship, compared to the more traditional phase-angle controller characteristic.

\section{EXPERIMENTAL VERIFICATION OF THE SMALL Signal ANALYSIS METHODOLOGY}

Fig. 9 shows experimental measurements of the small-signal frequency response of the converter along with those from the proposed analysis (42). The power factor is perturbed around $P F_{\text {bias }}=0.75\left(f_{s} \approx 157 \mathrm{kHz}, f_{s} \approx 161 \mathrm{kHz}\right.$ with $R_{L}=10 \Omega$, $R_{L}=20 \Omega$, respectively) through use of the self-oscillating power-factor controller considered previously. In order to appropriately consider the results a note about scaling is in order.
When $R_{L}=10 \Omega$, the converter gain at resonance is 0.674 , hence, with the specified input voltage, $V_{\text {out }}=12.1 \mathrm{~V}$, implying a steady-state gain of $+21.7 \mathrm{~dB}$ at $0 \mathrm{~Hz}$ between PF and $V_{\text {out }}$. Similarly when $R_{L}=20 \Omega$, the converter gain at resonance is 1.26 , hence, with the specified input voltage, $V_{\text {out }}=22.7 \mathrm{~V}$, giving a steady-state gain of $+27.1 \mathrm{~dB}$ at $0 \mathrm{~Hz}$ between PF and $V_{\text {out }}$. It can be seen that, in general, very good agreement between the results is seen to exist. Moreover, from Fig. 10 it can be seen that both the simulated and experimental output voltage dynamics closely follow a scaled version of the input power factor, thereby further demonstrating the controller's linear behavior, and, although the underlying state-variable model is eighth-order, the system is dominantly third-order. Furthermore, since the small-signal response is found to be constant across the full steady-state power-factor range, the small-signal response can also be considered to provide a good approximation of the large-signal behavior.

The model is shown to be experimentally validated up to $\sim 2 \mathrm{kHz}$, which is more than sufficient for the robust design of an outer output-voltage/current regulation controller. Typically, the output-regulation control bandwidth for resonant converters is designed to be smaller than that of the converter output-filter ( $3.4 \mathrm{kHz}$ in the prototype converter), which, by necessity, has a much lower bandwidth than the switching frequency. Nevertheless, for completeness, results from detailed time-domain nonlinear circuit simulations are also included at higher frequencies to further show the validity of the model.

\section{SUPPly-VolTAGE DistuRbance REJECTION}

To demonstrate the supply voltage disturbance rejection properties, the converter (detailed in Section VII) is initially excited such that the power factor is controlled to be $\mathrm{PF}=0.8$. Then, intermittently, the product $V_{\mathrm{dc}} \mathrm{PF}$ is controlled to be maintained at $V_{\mathrm{dc}} \mathrm{PF}=14.2$ through use of feed-forward action. With a constant dc supply voltage of $18 \mathrm{~V}$ both strategies provide $V_{\text {out }}=$ $9 \mathrm{~V}$ output when applied across a $10 \Omega$ load. Fig. 11 shows the ability of the latter method to provide improved regulation characteristics when $V_{\mathrm{dc}}=18+2 \sin (2 \pi 100 t)$-the choice of 


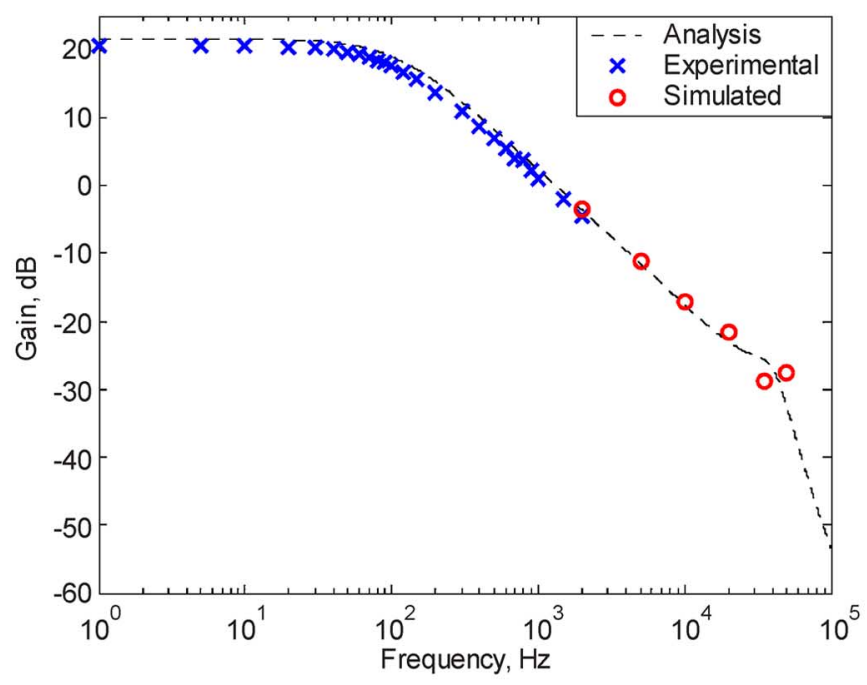

(a)

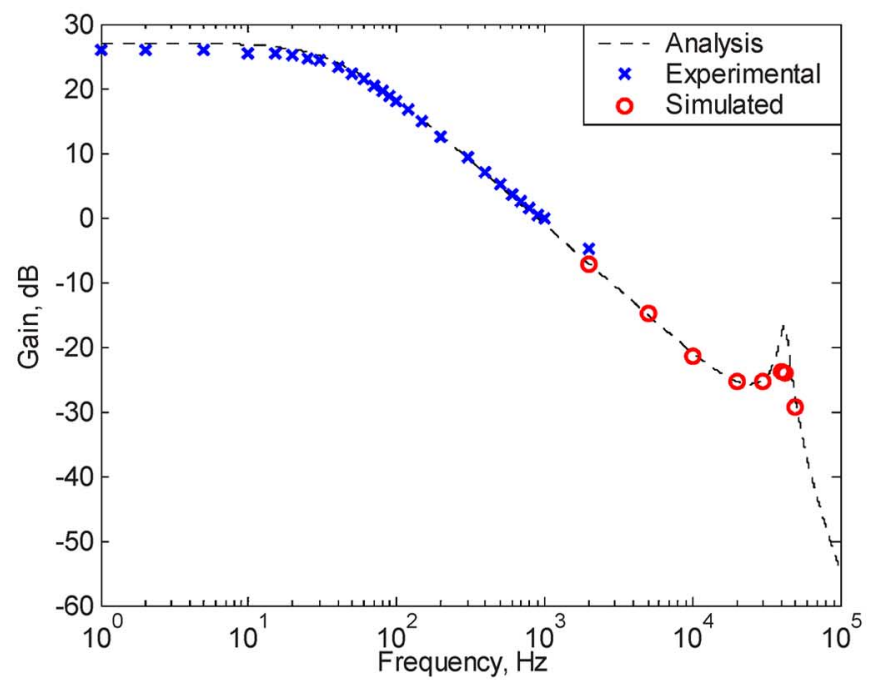

(a)

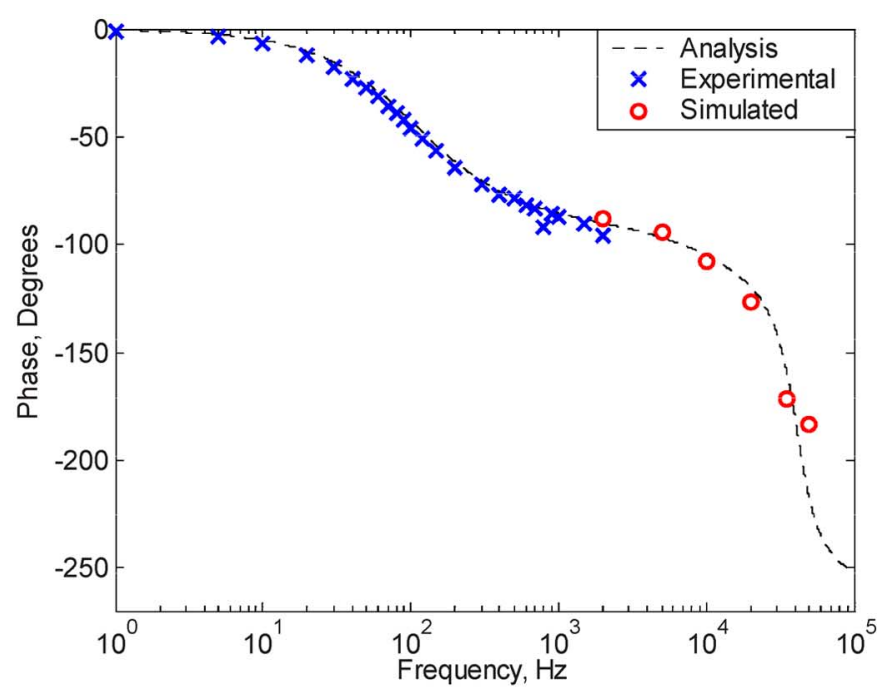

(b)

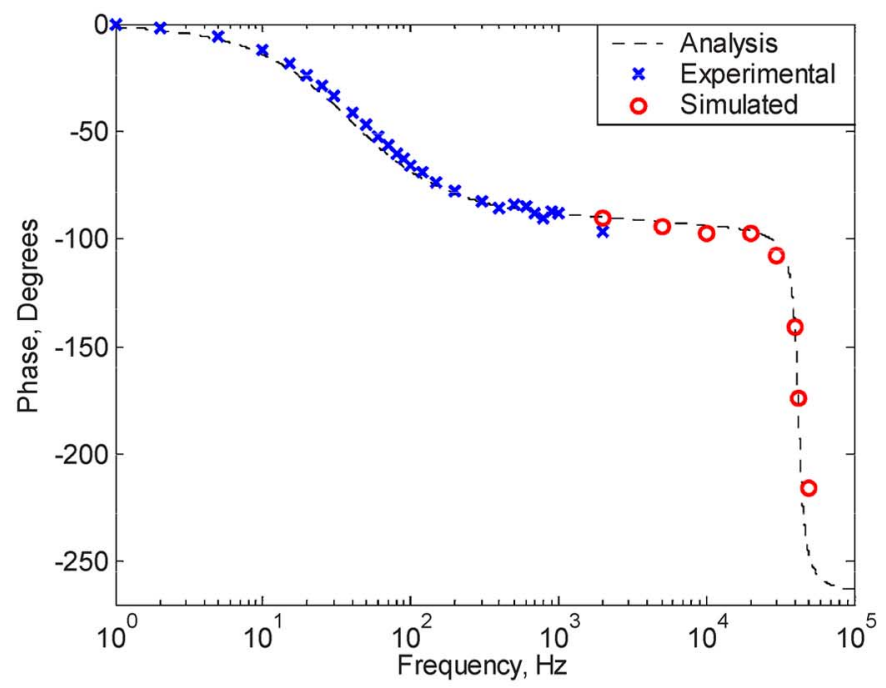

(b)

Fig. 9. Small-signal frequency response comparison between proposed analysis and experimental results for $10 \Omega$ load (a-b) and $20 \Omega$ load (c-d).

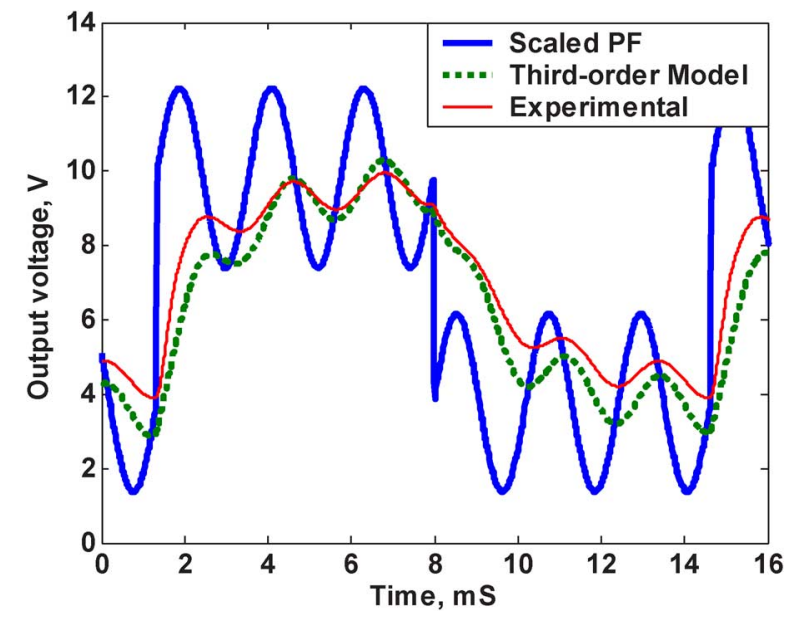

Fig. 10. Comparison between experimental and small-signal model response for $R_{L}=10 \Omega$ during a perturbed step change in power factor from $\mathrm{PF}=$ 0.8 to 0.3 . Note that a third-order numerically reduced model is utilized for the small-signal response. The power factor is scaled by $12.1 \mathrm{~V}$ since when the power-factor is unity the theoretical ideal output-voltage of the converter is $12.1 \mathrm{~V}$.
$100 \mathrm{~Hz}$ disturbance reflects the use of a full-bridge mains rectifier at the input to the converter, since the effects of higher frequency perturbations are attenuated due to the presence of the output filter and tank. In particular, it can be seen that during constant power-factor operation, the peak-peak output voltage ripple $(1.66 \mathrm{~V})$ is much larger than that obtained when utilizing feed-forward control action $(0.26 \mathrm{~V})$, thereby demonstrating the potential for improving regulation performance (see Fig. 12).

\section{Closed-Loop Output Voltage Regulation}

When power factor control is combined with the described feed-forward action (giving the equivalence of input voltage control) the approximate large-signal model (42) requires minor modification. Specifically, the supply voltage in (42) is removed from the $\mathbf{B}$ matrix and included in the input $\mathbf{u}$, giving

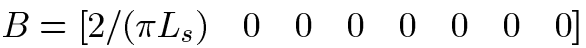

$$
\begin{aligned}
& u=V_{\mathrm{dc}} P F \text {. }
\end{aligned}
$$




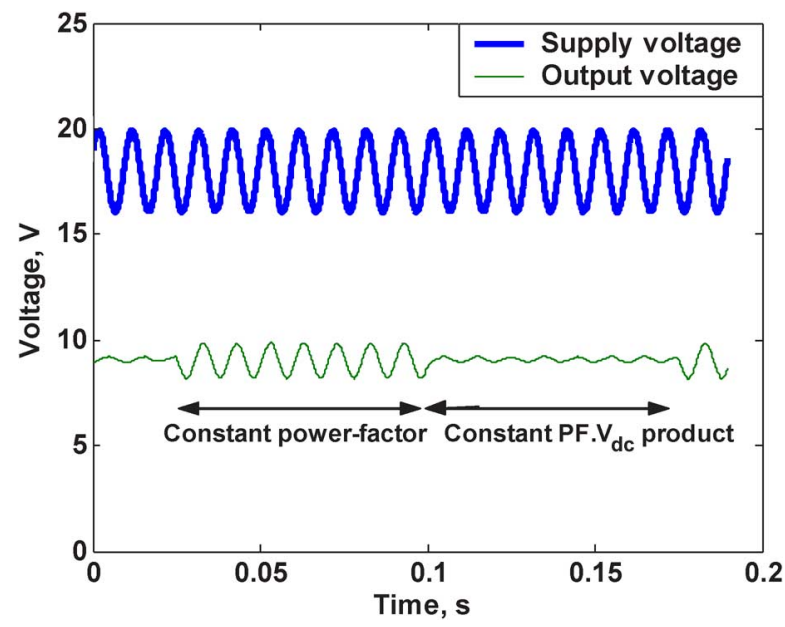

Fig. 11. Supply-voltage rejection with and without feed-forward action.

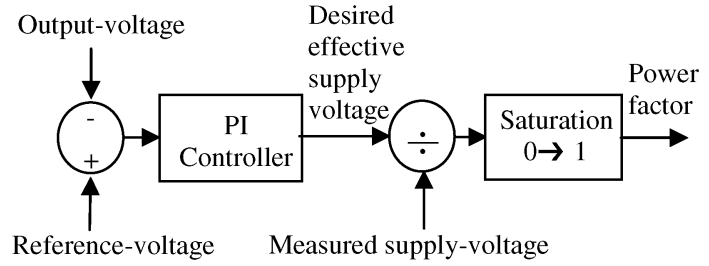

Fig. 12. Closed loop output voltage regulation with additional feed forward control.

TABLE I

CONTROLLer Gains AND AsSOCIATED 100- $\Omega$ LOAD GAIN/PhASE MARGINS

\begin{tabular}{|c|l|}
\hline Controller Gains & Gain/Phase Margins \\
\hline$K_{i}=1 \times 10^{3} K_{p}=2$ & $G M=9.25 P M=57.4^{\circ}$ \\
$K_{i}=1 \times 10^{3} K_{p}=10$ & $G M=1.85 P M=88.8^{\circ}$ \\
$K_{i}=10 \times 10^{3} K_{p}=2$ & $G M=9.24 P M=20.3^{\circ}$ \\
$K_{i}=10 \times 10^{3} K_{p}=10$ & $G M=1.85 P M=71.8^{\circ}$ \\
\hline
\end{tabular}

For output voltage control, the overall system will take a form similar to that shown in Fig. 12.

For stability, the loop-gain must never exceed unity magnitude when the phase shift crosses $-180^{\circ}$, for all possible load conditions. The loop-gain is found from

$$
L(s)=G(s) F(s)
$$

where $G(s)$ is the third-order reduced model of the converter and $F(s)$ is the controller model. The converter is to regulate the output voltage across a load ranging from 10's $\Omega$ to $100 \Omega$. For robustness, the controller gains are selected such that the converter remains stable with a phase margin $>20^{\circ}$, with an applied load of $100 \Omega$.

Implementing the control gains in Table I on the prototype converter, and stepping the load between $10 \Omega$ and $20 \Omega$, at $20 \mathrm{~Hz}$, with an electronic load, while regulating the outputvoltage at $9 \mathrm{~V}$ gives the responses presented in Figs. 13 and 14, for the two cases, respectively.

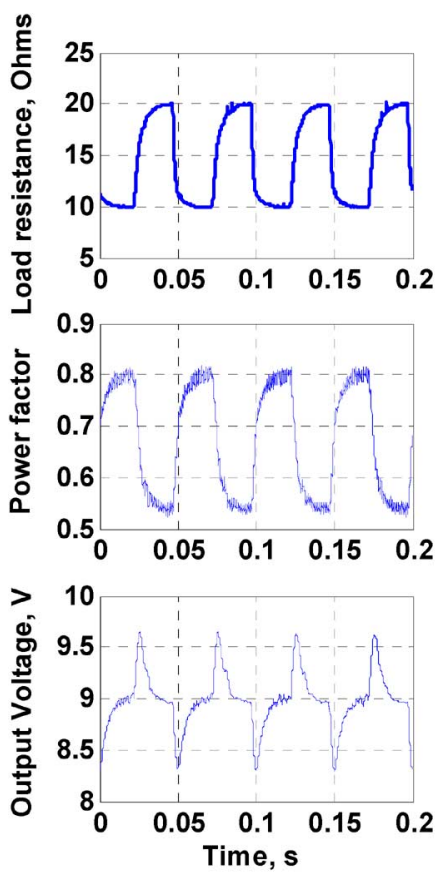

(a)
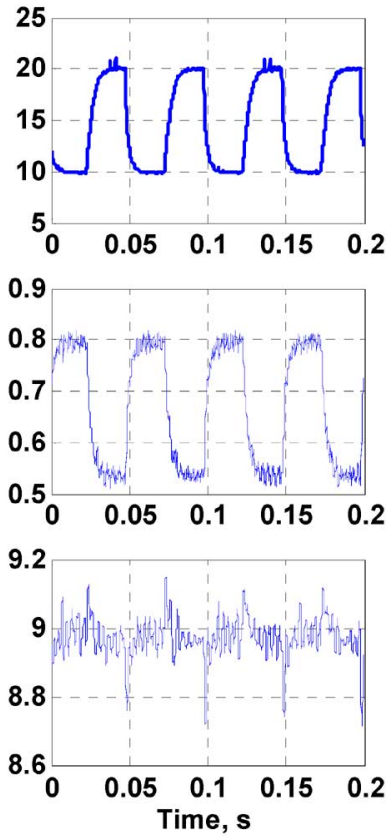

(b)
Fig. 13. Closed loop output-voltage regulation waveforms (a) $K_{p}=2 K_{i}=$ $1 \times 10^{3}$ and (b) $K_{p}=2 K_{i}=10 \times 10^{3}$.
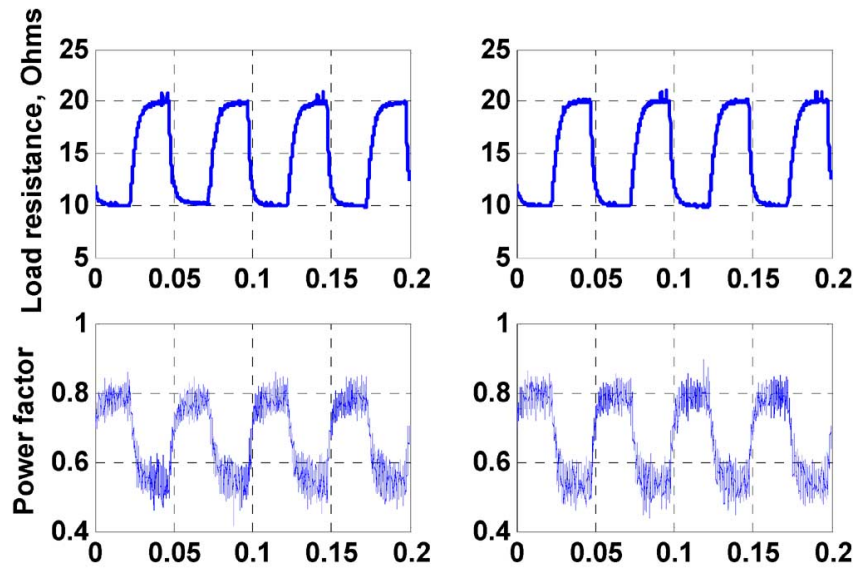

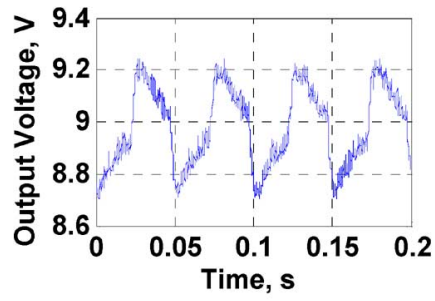

(a)

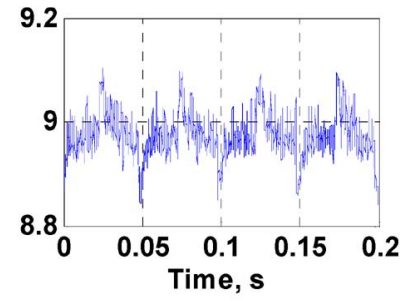

(b)
Fig. 14. Closed loop output-voltage regulation waveforms (a) $K_{p}=10 K_{i}=$ $1 \times 10^{3}$ and (b) $K_{p}=10 K_{i}=10 \times 10^{3}$.

It can be seen that the output-voltage regulation is being obtained in each case in response to a relatively aggressive disturbance stimuli. The converter can also be operated as a constant current-source by feeding back a measurement of output current instead of the output voltage. By way of example, for a demand current of $0.75 \mathrm{~A}$, the resulting closed loop responses shown in Fig. 15 are obtained. Controller gains of $K_{p}=15$ 

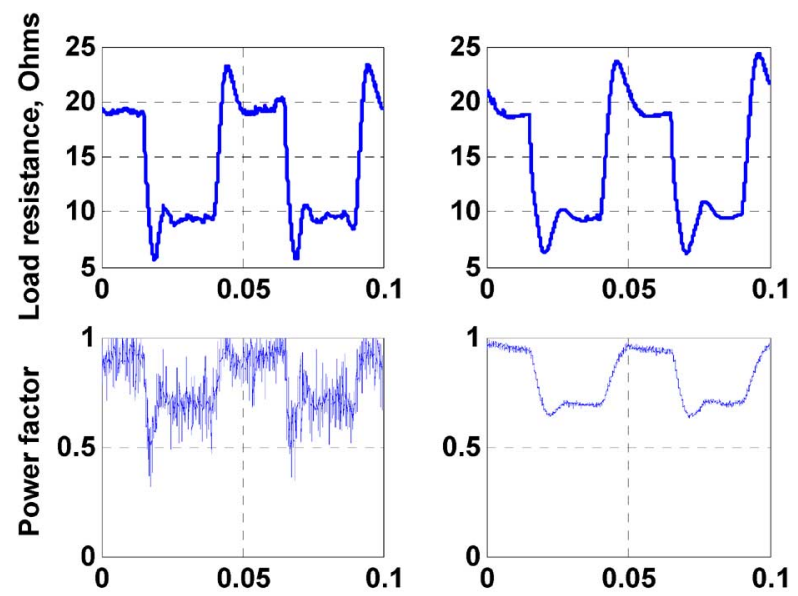

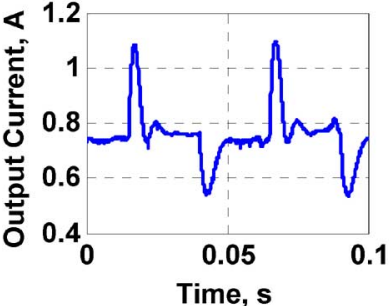

(a)

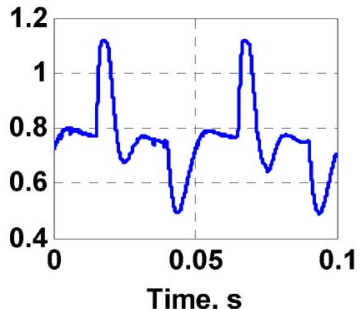

(b)
Fig. 15. Closed loop output current control (a) $K_{p}=15 K_{i}=2.5 \times 10^{3}$ and (b) $K_{p}=1 K_{i}=2.5 \times 10^{3}$.

$K_{i}=2.5 \times 10^{3}$ and $K_{p}=1 K_{i}=2.5 \times 10^{3}$ are used, respectively, in Fig. 15(a) and (b). Again, it can be seen that regulation is clearly evident in response to the aggressive stimuli. However, notably, significant overshoot is present. This is a common feature of such systems and is a consequence of the power flow to the output filter being unidirectional-the controller does not have the capability to remove stored energy, and relies on the load depleting the charge on the filter capacitor.

\section{Alternative Switching Techniques}

Two alternative switching techniques are shown in Fig. 16, each having the advantage that a switching event occurs on a zero-current crossing point, thereby facilitating reduced switching losses. When employing the fixed falling-edge method, shown in Fig. 16(a), the magnitude of the fundamental of the voltage, normalized to that for a duty-cycle of $50 \%$ is

$$
G_{1}(\alpha)=\cos \left(\frac{\alpha}{2}\right)
$$

where $\alpha$ is the phase-angle between the rising-edge of the switching voltage and the rising zero-crossing of the sinusoidal current. (i.e., when $\alpha=0$ and $\alpha=\pi$, the switching-waveform is, respectively, square wave and $\mathrm{dc}$ ). The fundamental of the switching-voltage is, in general, out of phase with the sinusoidal current. The fraction of switching voltage fundamental component that can be considered as being in-phase with the current-waveform, is

$$
G_{2}(\alpha)=\cos \left(\frac{\alpha}{2}\right)
$$

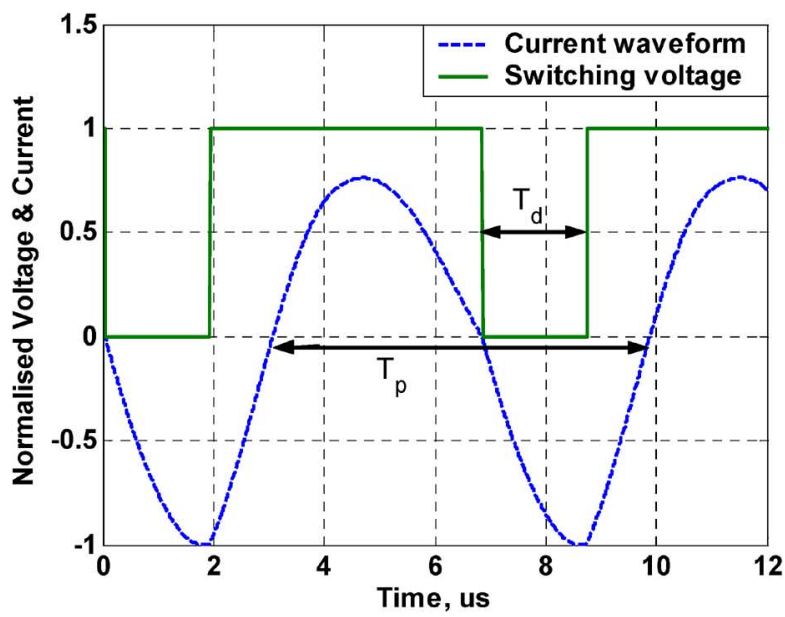

(a)

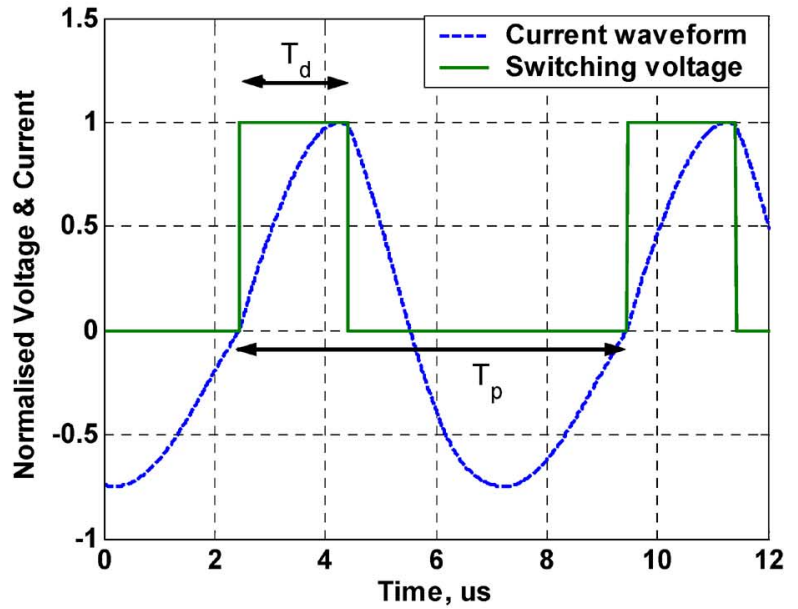

(b)

Fig. 16. Alternative self-oscillating switching strategies-series inductor current zero-crossing fixed: (a) falling-edge and (b) rising-edge.

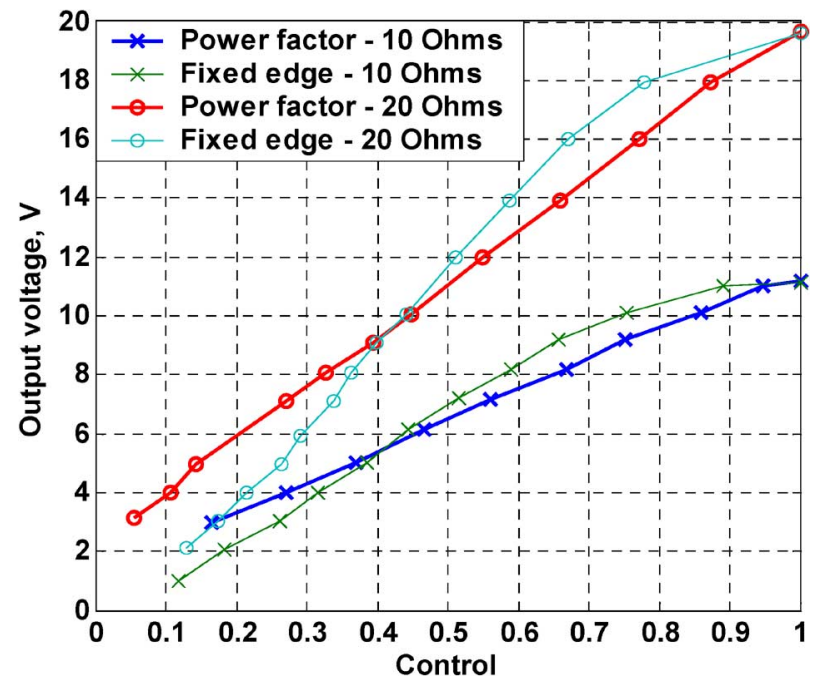

Fig. 17. Comparison between control input and output-voltage for two different loads under power-factor control and the alternative fixed rising-edge control.

[note that $\alpha / 2$ is the phase shift between the fundamental of the switching voltage and current waveform]. 


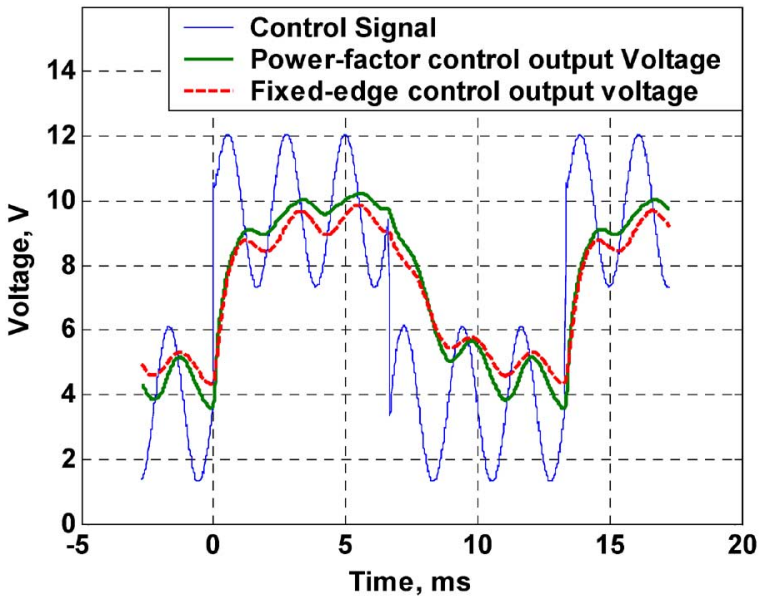

Fig. 18. Output voltage as control signal is varied for both power-factor and fixed edge control with an applied $10 \Omega$ load.

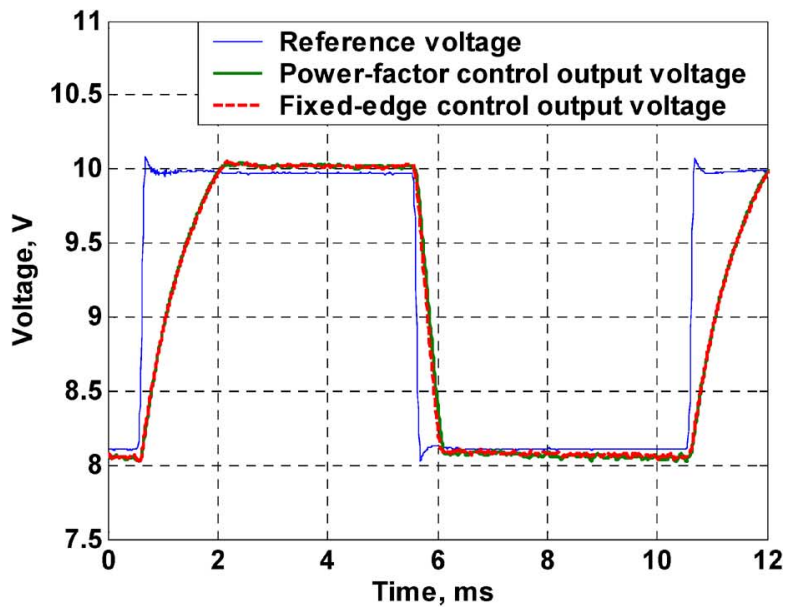

(a)

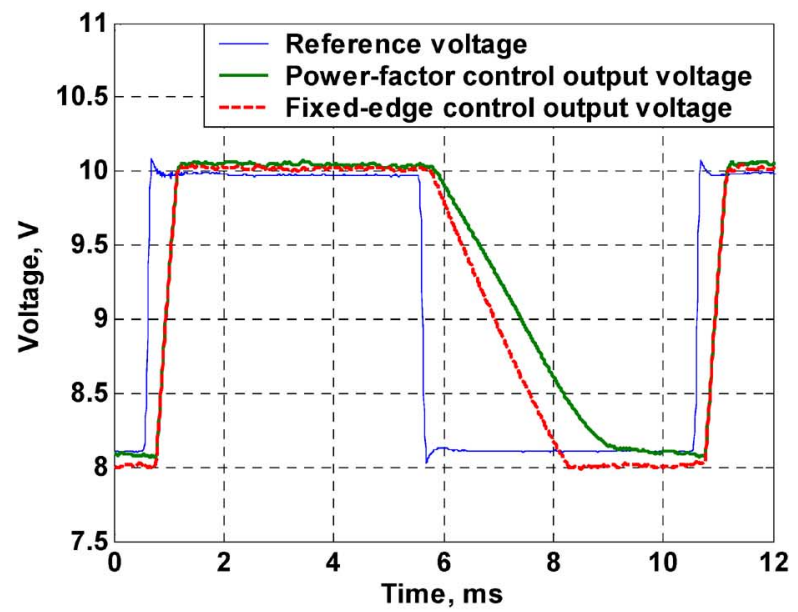

(b)

Fig. 19. Closed loop output voltage regulation: (a) $10 \Omega$ load and (b) $50 \Omega$ load. The reference voltage is stepped between $8 \mathrm{~V}$ and $10 \mathrm{~V}$ at $100 \mathrm{~Hz}$.

The overall switching-voltage component that is in-phase with the sinusoidal current-waveform, normalized to the condition when the converter is switched with a $50 \%$ duty cycle, at resonance, is

$$
G=G_{1}(\alpha) G_{2}(\alpha)=\cos \left(\frac{\alpha}{2}\right)^{2} .
$$

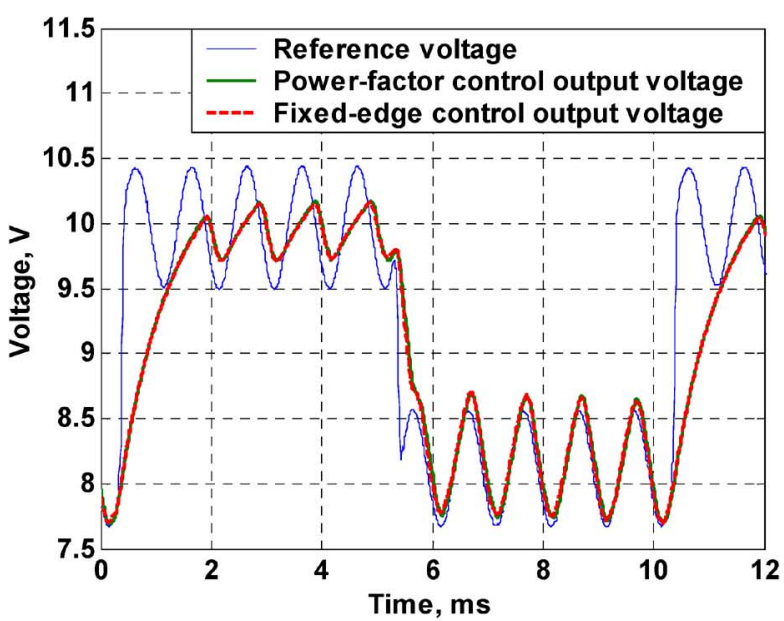

(a)

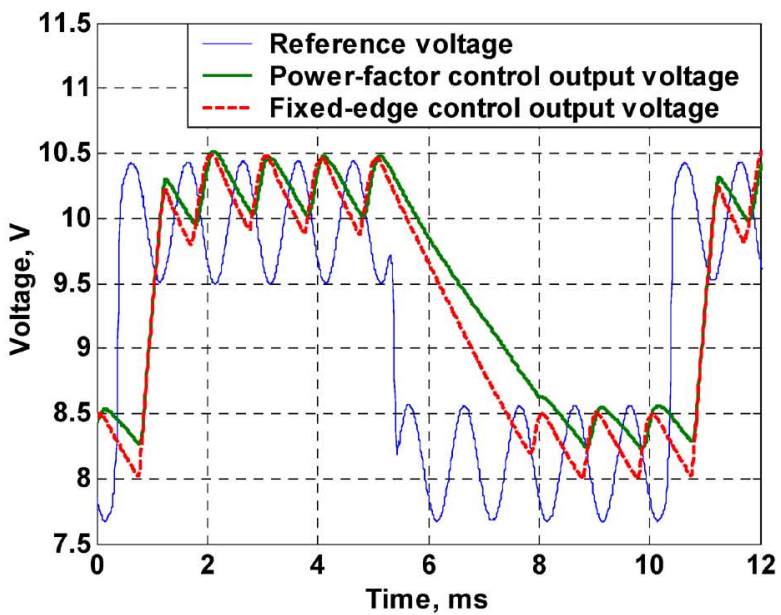

(b)

Fig. 20. Closed loop output voltage regulation (a) $10-\Omega$ load and (b) $50-\Omega$ load. The reference voltage is stepped between 8 and $10 \mathrm{~V}$ at $100 \mathrm{~Hz}$ with a $1-\mathrm{kHz}$ $0.5-\mathrm{V}$ amplitude perturbation.

The time $T_{d}$ is therefore calculated as follows:

$$
T_{d}=T_{p}\left(\frac{1}{2}-\frac{\cos ^{-1}(G)}{\pi}\right) .
$$

From Fig. 16 it can be seen that the current waveforms are asymmetric about the positive and negative half-cycles, and are therefore not sinusoidal, which was an underlying assumption for the analysis. Simulation studies have shown that, generally, (50) does not provide a sufficiently accurate linearization mechanism for the falling-edge switching strategy, similarly when an equivalent methodology is applied to the rising-edge technique, the resulting behavior is found to be alike.

The fixed rising-edge strategy is therefore tested without the use of any linearization equation, thereby eliminating the need for a lookup table within the FPGA, and the delay time $T_{d}$ is given simply by

$$
T_{d}=T_{p} \frac{G}{2}
$$

i.e when $G=1$, the delay time $T_{d}$ is a half-period, giving a square-wave switching voltage in phase with the current waveform. When $G=0$, no switching occurs and the input voltage is 


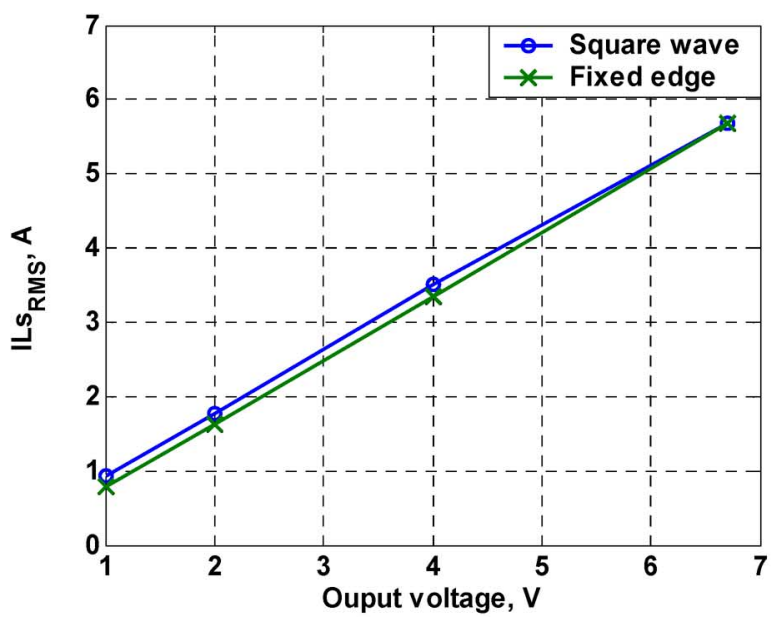

(a)

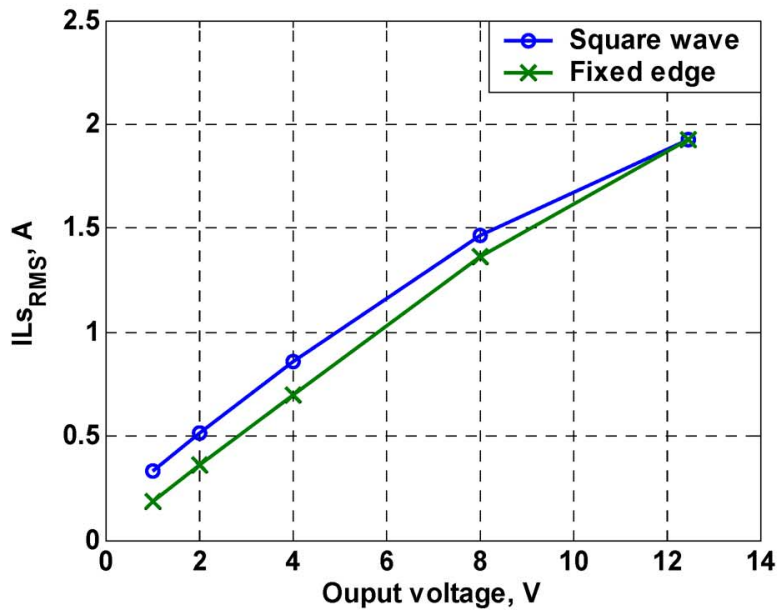

(b)

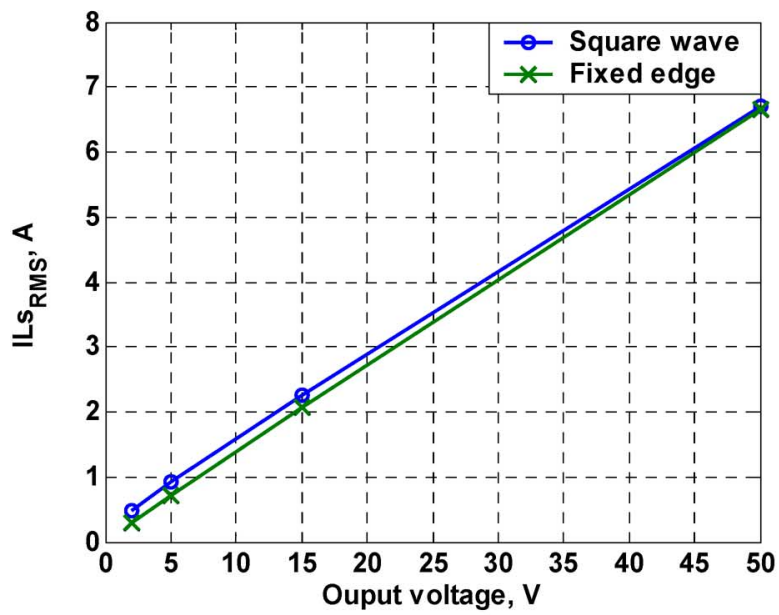

(c)

Fig. 21. Simulated RMS series-inductor current and switching frequency for an applied load of: (a) $1 \Omega$, (b) $10 \Omega$, and (c) $50 \Omega$ across a range of output voltages when utilizing power-factor (square-wave) and fixed-edge control.

clamped to $0 \mathrm{~V}$. Fig. 16 provides a comparison of the converter output voltage for various loads, as the control input is varied, during power-factor and fixed rising-edge control.

From Fig. 17 it can be seen that the fixed-edge methodology provides approximately linear behavior. The large-signal model for power-factor self-oscillating control, approximated by the small-signal model presented in Section IV, may therefore be
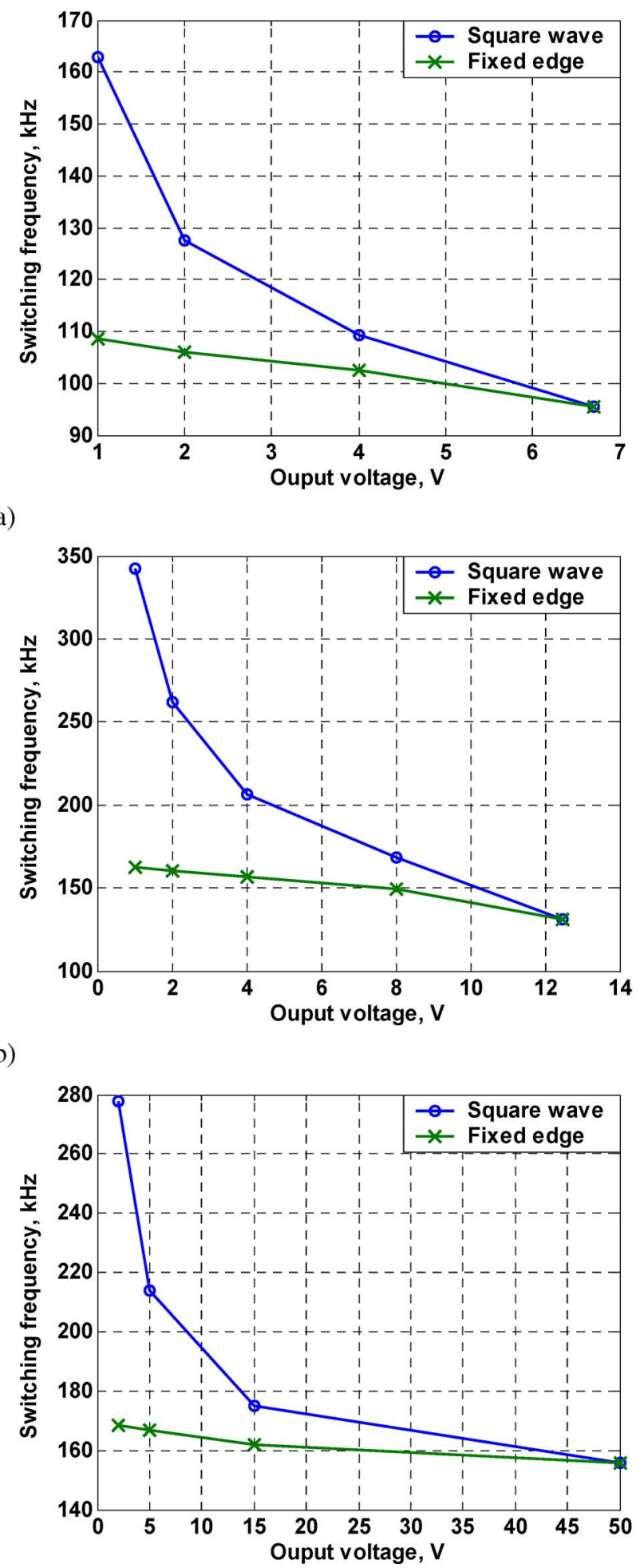

considered to provide a reasonable large-signal model for the fixed rising-edge control scheme.

Fig. 18 compares the converter output-voltage and control-input for both power-factor control and fixed-edge control. Both methods result in similar control signal to output-voltage characteristics, and, for control purposes the power-factor large-signal model may be sufficient. 


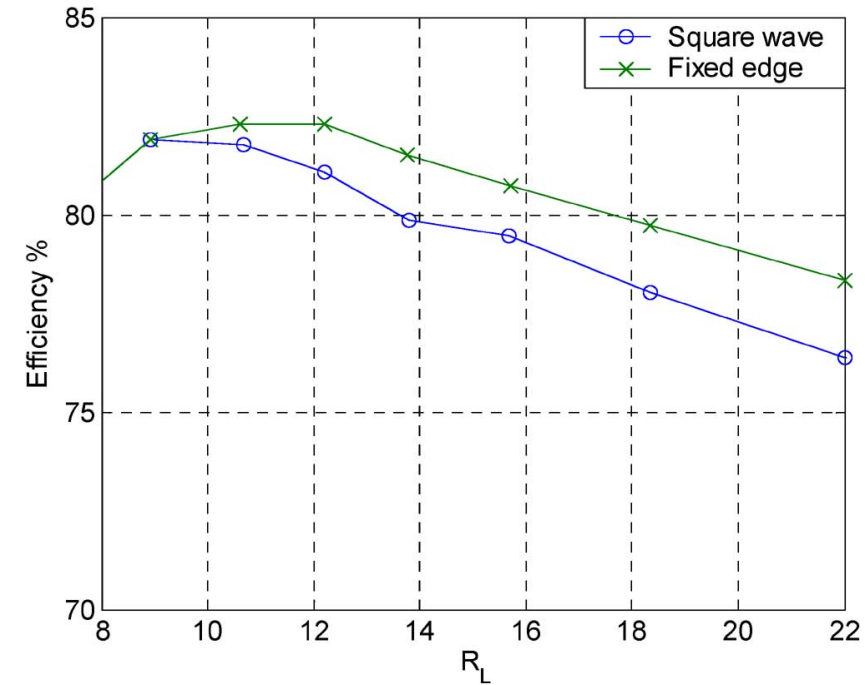

Fig. 22. Efficiency comparison between power-factor and fixed rising-edge (no lookup table) control when output current is regulated at $1 \mathrm{~A}$ across a wide load range.

Closing the loop with $K_{p}=30$ and $K_{i}=1 \times 10^{-3}$, and comparing the converter output voltage for both methodologies as the output-voltage reference is varied gives the transient responses shown in Fig. 19.

Comparing Fig. 19(a) with Fig. 19(b) it can be seen that the output-voltage fall-time, for the case of the $50 \Omega$ load, is much slower than that applied across the $10-\Omega$ load, demonstrating the load dependent control dynamics discussed previously. Conversely, the output-voltage rise-time for the $50-\Omega$ load case is much faster. This is a consequence of the controller demanding a transiently higher voltage than the desired $10 \mathrm{~V}$, with the converter capable of developing approximately $50 \mathrm{~V}$ at resonance with a $50-\Omega$ load in this case.

A similar effect can be seen in Fig. 20(a). The demand and output-voltages are very similar when the demand is less than $9 \mathrm{~V}$, and less so when higher voltages are demanded.

Of note, is the use of the 'fixed rising-edge methodology' provides the qualities of a much smaller RMS series-inductor current, and a reduced switching bandwidth — both of which act to improve efficiency. This is demonstrated in Fig. 21 where the RMS series-inductor current and switching frequency, are shown for both control methodologies [Note: the term "Squarewave" in Figs. 21 and 22 is used to represent any control technique providing a square-wave excitation voltage $\left(V_{s}\right)$ to the input of the resonant tank (as in the case of power-factor control, or the traditional variable frequency control)].

When regulating the output-current at $0.5 \mathrm{~A}$, into a load varying between $10 \Omega \rightarrow 50 \Omega$, the switching bandwidth required for 'square-wave' excitation-voltage techniques is $170 \mathrm{kHz} \rightarrow 190 \mathrm{kHz}$, a substantially greater range than the $156 \mathrm{kHz} \rightarrow 160 \mathrm{kHz}$ required when utilizing the "fixed-edge" technique.

Fig. 22 compares the prototype's (without synchronous rectification) closed-loop converter efficiency as the output current is regulated at $1 \mathrm{~A}$ over a range of applied loads, utilizing both the power-factor ("square wave") and fixed rising-edge control methodologies. From the results it can be seen that utilization of the "fixed edge" scheme provides a higher efficiency solution as a consequence of switching events occurring at a zero-current crossing, reduced RMS series-inductor current (as a result of the reduced excitation waveform duty-cycle), and switching close to resonance across the entire output-voltage range (since the system is forced to commutate in phase with the resonant current).

\section{CONCLUSION}

A control strategy is provided for the LCC current-output resonant converter that is ultimately, more widely applicable to other resonant converter topologies. The methodology is advantageous in that it initially linearizes the steady-state input-to-output conversion ratio and, secondly, the large-signal converter dynamics become predominantly third-order. At present, no other reported control methodologies exhibit such simple linear behavior. Through the well-defined behavior of the power-factor controlled converter, the design of an additional controller to obtain closed-loop output-voltage/current regulation is greatly simplified, allowing robust and/or well documented adaptive control methods to be applied. This, therefore, addresses one of the main drawbacks for the use of resonant converters viz. the lack of literature on robust control of such systems. A further switching scheme is also proposed with reduced switching bandwidth, RMS series-inductor current, and approximately similar large signal dynamics to that of a power-factor controller converter.

\section{REFERENCES}

[1] R. L. Steigerwald, "A comparison of half-bridge resonant converter topologies," IEEE Trans. Power Electron., vol. PE-3, no. 2, pp. 174-182, Apr. 1988.

[2] M. K. Kazimierczuk and D. Czarkowski, Resonant Power Converters. New York: Wiley, 1995, ch. 8 \& 17.

[3] M. Castilla, L. Garcia de Vicuna, J. M. Guerrero, J. Matas, and J. Miret, "Sliding-mode control of quantum series-parallel resonant converters via input-output linearization," IEEE Trans. Ind. Electron., vol. 52, no. 2, pp. 566-575, Apr. 2005.

[4] A. J. Forsyth and Y. K. E. Ho, "High performance control of the seriesparallel resonant converter," Proc. Inst. Elect. Eng., vol. 144, no. 2, pp. 131-139, Mar. 1997.

[5] Y. Yin and R. Zane, "Digital phase control for resonant inverters," IEEE Power Electron. Lett., vol. 2, no. 2, pp. 51-53, Jun. 2004.

[6] E. X. Yang, F. C. Lee, and M. M. Jovanovic, "Small-signal modeling of LCC resonant converter," in Proc. Power Electron. Spec. Conf., 1992, pp. 941-948.

[7] M. Castilla, L. G. de Vicuna, M. Lopez, and V. Barcons, "An averaged large-signal modelling method for resonant converters," in Proc. 23 rd Int. Conf. Ind. Electron., Control Instrum. (IECON'97), Nov. 9-14, 1997, vol. 2, pp. 444-452.

[8] Y. Yin, R. Zane, R. Erickson, and J. Glaser, "Dynamic analysis of frequency-controlled electronic ballasts," in Proc. 37th IAS Annu. Meeting Ind. Appl. Conf., Oct. 13-18, 2002, vol. 1, pp. 685-669.

[9] C. T. Rim and G. H. Cho, "Phasor transformation and its application to the DC/AC analyses of frequency phase-controlled Series Resonant Converters (SRC)," IEEE Trans. Power Electron., vol. 5, no. 2, pp. 201-211, Apr. 1990.

[10] S. Ben-Yaakov, S. Glozman, and R. Rabinovici, "Envelope simulation by SPICE-compatible models of electric circuits driven by modulated signals," IEEE Trans. Ind. Electron., vol. 47, no. 1, pp. 222-225, Feb. 2000.

[11] A. J. Gilbert, D. A. Stone, and C. M. Bingham, "Rapid design of LCC current-output resonant converters with reduced electrical stresses," in Proc. Eur. Conf. Power Electron. Appl., Sep. 11-14, 2005, pp. 1-9.

[12] A. J. Gilbert, D. A. Stone, and C. M. Bingham, "Design of an LCC current-output resonant converter for use as a constant current source," in Proc. Eur. Conf. Power Electron. Appl., Sep. 2-5, 2007, pp. 1-6. 


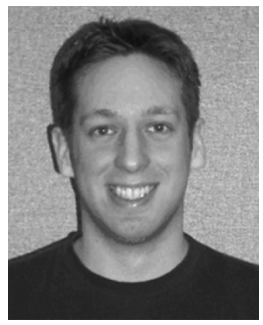

Adam J. Gilbert received the M.Eng. and Ph.D. degrees in electronic and electrical engineering from The University of Sheffield, Sheffield, U.K., in 2003 and 2008, respectively.

His research interests currently include the modelling and control of switching power converters with particular emphasis on high-efficiency resonant supplies.

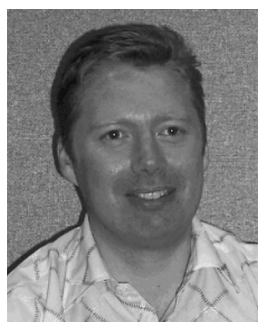

Christopher M. Bingham received the B.Eng degree in electronic systems and control engineering from Sheffield City Polytechnic, Sheffield, U.K., in 1989, the M.Sc.(Eng) degree in control systems engineering from the University of Sheffield, Sheffield, U.K., in 1990, and the Ph.D. degree in control systems to accommodate nonlinear dynamic effects in aerospace flight-surface actuators from Cranfield University, Bedfordshire, U.K., in 1994.

He was a Post-Doctoral Researcher at Cranfield University, until subsequently taking up a research position at the University of Sheffield. Since 1998, he has been a Lecturer in the Department of Electronic and Electrical Engineering, University of Sheffield. His current research interests include traction control/anti-lock braking systems for electric vehicles, electromechanical actuation of flight control surfaces, control of active magnetic bearings for high-speed machines, sensorless control of brushless machines, analysis and design of resonant converter systems, and the control of high-performance UAVs.

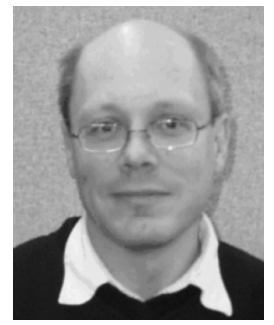

David A. Stone received the B.Eng. degree in electronic engineering from the University of Sheffield, Sheffield, U.K., in 1984 and the Ph.D. degree from Liverpool University, Liverpool, U.K., in 1989.

He returned to the University of Sheffield as a member of academic staff specializing in power electronics and machine drive systems. His current research interests are in hybrid-electric vehicles, battery charging, EMC, and novel lamp ballasts for low pressure fluorescent lamps.

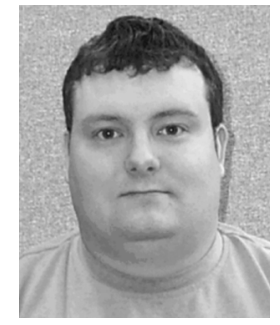

Martin P. Foster received the B.Eng. degree in electronic and electrical engineering, the M.Sc.(Eng) degree in control systems, and the Ph.D. degree in rapid analysis and design of resonant converters from the University of Sheffield, Sheffield, U.K., in 1998, 2000, and 2003, respectively.

His current research interests include the modeling and control of switching power converter with particular emphasis on high-efficiency resonant supplies, and novel control methods for autonomous aerospace vehicles. 